\title{
TREM-1 orchestrates angiotensin II-induced monocyte trafficking and promotes experimental abdominal aortic aneurysm
}

\author{
Marie Vandestienne, ${ }^{1}$ Yujiao Zhang, ${ }^{1}$ Icia Santos-Zas, ${ }^{1}$ Rida AI-Rifai, ${ }^{1}$ Jeremie Joffre, ${ }^{1}$ Andreas Giraud, ${ }^{1}$ Ludivine Laurans, ${ }^{1}$ \\ Bruno Esposito, ${ }^{1}$ Florence Pinet, ${ }^{2}$ Patrick Bruneval, ${ }^{1,3}$ Juliette Raffort, ${ }^{4}$ Fabien Lareyre, ${ }^{4}$ Jose Vilar, ${ }^{1}$ Amir Boufenzer, ${ }^{5}$ \\ Lea Guyonnet, ${ }^{6,7,8}$ Coralie Guerin, ${ }^{6,7,8}$ Eric Clauser, ${ }^{1}$ Jean-Sébastien Silvestre, ${ }^{1}$ Sylvie Lang, ${ }^{9}$ Laurie Soulat-Dufour, ${ }^{9}$ \\ Alain Tedgui, ${ }^{1}$ Ziad Mallat, ${ }^{1,10}$ Soraya Taleb, ${ }^{1}$ Alexandre Boissonnas, ${ }^{11}$ Marc Derive, ${ }^{5}$ Giulia Chinetti, ${ }^{4}$ and Hafid Ait-Oufella ${ }^{1,12}$ \\ 'Université de Paris, Inserm U970, Paris-Cardiovascular Research Center, Paris, France. ${ }^{2}$ INSERM U1167-Institut Pasteur de Lille, Lille, France. ${ }^{3}$ Department of Anatomopathology, Hôpital Européen Ceorges \\ Pompidou, Assistance Publique-Hôpitaux de Paris (AP-HP), Paris, France. ${ }^{4}$ Université Côte d'Azur, Centre Hospitalo-Universitaire (CHU), INSERM, C3M, Nice, France. ${ }^{5}$ INOTREM SA, Nancy, France. ${ }^{6}$ Université \\ de Paris, Innovative Therapies in Haemostasis, INSERM, F-75006, Paris, France. ${ }^{7}$ Institut Curie, Cytometry Platform F-75006, Paris, France. ${ }^{8}$ Department of Infection and Immunity, Luxembourg Institute \\ of Health, Luxembourg, Luxembourg. ${ }^{9}$ Cardiology Department, Hôpital Saint-Antoine, AP-HP, Sorbonne Université, Paris, France. ${ }^{10}$ Department of Medicine, Division of Cardiovascular Medicine, University \\ of Cambridge, Cambridge, United Kingdom. " 1 Sorbonne Université, INSERM, CNRS, Centre d'Immunologie et des Maladies Infectieuses Cimi-Paris, Paris, France. ${ }^{12}$ Medical Intensive Care Unit, Hôpital Saint- \\ Antoine, AP-HP, Sorbonne Université, Paris, France.
}

\begin{abstract}
The triggering receptor expressed on myeloid cells 1 (TREM-1) drives inflammatory responses in several cardiovascular diseases but its role in abdominal aortic aneurysm (AAA) remains unknown. Our objective was to explore the role of TREM-1 in a mouse model of angiotensin II-induced (Angll-induced) AAA. TREM-1 expression was detected in mouse aortic aneurysm and colocalized with macrophages. Trem1 gene deletion ( $A p o e^{-/-T r e m 1^{-/-}}$), as well as TREM-1 pharmacological blockade with LR-12 peptide, limited both AAA development and severity. Trem1 gene deletion attenuated the inflammatory response in the aorta, with a reduction of $111 b$, Tnfa, Mmp2, and Mmp9 mRNA expression, and led to a decreased macrophage content due to a reduction of Ly6Chi classical monocyte trafficking. Conversely, antibody-mediated TREM-1 stimulation exacerbated $\mathrm{Ly}_{6 \mathrm{C}} \mathrm{Ch}^{\mathrm{hi}}$ monocyte aorta infiltration after Angll infusion through $\mathrm{CD} 62 \mathrm{~L}$ upregulation and promoted proinflammatory signature in the aorta, resulting in worsening AAA severity. Angll infusion stimulated TREM-1 expression and activation on Ly6 $\mathrm{C}^{\mathrm{hi}}$ monocytes through Angll receptor type I (AT $\mathrm{R}$ ). In human AAA, TREM-1 was detected and TREM1 mRNA expression correlated with SELL mRNA expression. Finally, circulating levels of sTREM-1 were increased in patients with AAA when compared with patients without AAA. In conclusion, TREM-1 is involved in AAA pathophysiology and may represent a promising therapeutic target in humans.
\end{abstract}

\section{Introduction}

Nonsyndromic abdominal aortic aneurysm (AAA) is one of the leading causes of cardiovascular death, particularly in men and women over 60 years of age (1). Other defined risk factors associated with the development of AAA include male sexual phenotype, White race, a positive family history, smoking, and atherosclerosis. The mechanisms of AAA formation are complex; they include elastin degradation, collagen remodeling, smooth muscle cell apoptosis, and infiltration of inflammatory cells (2). During

Authorship note: YZ and ISZ contributed equally to the work. Conflict of interest: MD is cofounder of INOTREM, a company developing TREM-1 inhibitors. A Boufenzer, MD, and HAO applied a patent on the measurement of plasma STREM-1 concentration to predict outcome in cardiovascular diseases and applied for a patent on therapeutic strategies using inhibiting peptides derived from TREM-1 (patent no. W02014037565A2; Inhibiting peptides derived from triggering receptor expressed on myeloid cells-1 (trem-1) trem-like transcript 1 (tlt-1) and uses thereof). Copyright: (5) 2021, American Society for Clinical Investigation.

Submitted: July 23, 2020; Accepted: November 20, 2020; Published: January 19, 2021. Reference information: J Clin Invest. 2021;131(2):e142468.

https://doi.org/10.1172/JCl142468. the first critical steps of experimental AAA formation, circulating monocytes interact with activated endothelial cells and are recruited into the aortic wall in a biphasic manner. Ly6Chigh classical monocytes (Ly6C hi) dominate the acute phase of injury, whereas Ly6Clow nonclassical monocytes $\left(\right.$ Ly6 $\mathrm{C}^{\mathrm{lo}}$ ) are prevalent thereafter (3). Recruitment to the aortic wall partly depends on GM-CSF (4) and chemokines/chemokine receptors, including CCL2/ CCR2, CX3CL1/CX3CR1, and CCL-5/CCR5 axes (5), and adhesion molecules such as selectins. Monocyte differentiation into macrophages and activation are necessary steps for the formation of aneurysms, associated with the local release of inflammatory cytokines (TNF- $\alpha$, IL-1 $\beta$ ) and matrix metalloproteinases (MMPs), which induce arterial wall destruction, subsequent aorta dilatation, and life-threatening aneurysm rupture. Neutrophils present within the aneurysmal wall and the adjacent thrombus also contributes to AAA formation and complications (6). However, there is currently no specific treatment targeting the immunoinflammatory response in AAA and no antiinflammatory medical therapy is available to prevent excessive inflammatory remodeling of the abdominal aortic wall. 
Triggering receptor expressed on myeloid cells 1 (TREM-1) is a member of the immunoglobulin superfamily $(7,8)$ constitutively expressed by neutrophils and monocytes/macrophages. TREM-1 expression increases in response to lipopolysaccharide or other sterile pathogen-associated molecular patterns, following activation of Toll-like receptors and nucleotide-binding oligomerization domain-receptors $(8,9)$. Engagement of TREM-1, after association with the adapter protein DAP12, results in the production of proinflammatory cytokines, including TNF- $\alpha$ and IL- $1 \beta$, and chemokines, including IL-8, CCL-2, and CCL-7, along with rapid neutrophil degranulation (10). TREM-1 also regulates myeloid cell migration to inflammatory sites upon bacterial invasion $(8,11)$. The role of TREM-1 has been mainly investigated in septic shock, but previous studies showed that it is also critical in several sterile inflammation conditions, including atherosclerosis and postischemic myocardial remodeling (12). In a large multicenter cohort of patients with acute coronary syndromes, our group previously found that plasma levels of soluble TREM-1 were an independent predictor of major adverse cardiovascular events (12). Interestingly, LR12 peptide, a decoy receptor that strongly inhibits TREM-1 engagement by competing with its still unknown endogenous ligand, has been shown to modulate innate immune responses following bacterial aggression in rodents (13), pigs (14), and primates (15), resulting in an attenuation of organ dysfunction and improved outcome $(13,14)$. LR-12 treatment also reduces atherosclerosis development and limits deleterious postischemic cardiac remodeling in mice (12).

In a mouse model of AngII-induced AAA, we showed that both Trem 1 gene deletion and pharmacological blockade limit classical monocyte recruitment into the abdominal aorta and decrease AAA severity. Conversely, TREM-1 stimulation using a monoclonal anti-TREM-1 agonist antibody exacerbates monocyte infiltration in the aorta and worsens AAA severity. AngII increases TREM-1 expression in classical monocytes through AngII receptor type I ( $\left.\mathrm{AT}_{1} \mathrm{R}\right)$ engagement. Finally, we found that TREM-1 orchestrates monocyte mobilization from the spleen as well as aortic wall infiltration through CD62L upregulation. Our findings identify TREM- 1 as a new actor in the pathophysiology of AAA, which may represent a novel therapeutic target to combat AAA formation.

\section{Results}

Trem1 deficiency limits AAA development in AngII-infused Apoe ${ }^{-/-}$ mice. Given that TREM-1 is a key regulator of myeloid cell activity and that innate immunity plays a major role in AAA development, we investigated whether TREM-1 was involved in the pathophysiology of AAA. We used a well-validated experimental model of AAA based on AngII infusion in hypercholesterolemic Apoe ${ }^{-/-}$mice (16). TREM-1 expression was detected in the aorta of $A p o e^{-/-}$mice after AngII infusion at both mRNA (Figure 1A) and protein levels (Figure 1B), but not after PBS infusion. Using immunofluorescence staining, we showed that TREM- 1 colocalized with CD68 ${ }^{+}$ macrophages in the aortic wall (Figure 1B). In order to address the role of TREM-1 in the development of AngII-induced AAA, Apoe - $^{-1}$ $\mathrm{TremI}^{+/+}$and $\mathrm{Apoe}^{-/-} \mathrm{Trem}^{-/-}$mice were infused with AngII and key pathophysiological events associated with AAA development were assessed at different time points (Figure 1C) (16). The absence of TREM-1 expression on myeloid cells in $\mathrm{Apoe}^{-/-} \mathrm{Trem}^{-/-}$mice was confirmed by flow cytometry (Figure 1D and Supplemental Figure 1; supplemental material available online with this article; https:// doi.org/10.1172/JCI142468DS1). After 28 days of AngII infusion, abdominal aortic diameter was significantly lower in Apoe -Trem1 $^{-/-}$ mice as compared with control Apoe - $^{-/}$Trem $^{+/+}$mice $(1.2 \pm 0.2 \mathrm{~mm}$ vs $1.9 \pm 0.7 \mathrm{~mm}, P<0.05$ ) (Figure $1 \mathrm{E}$ ). AAA incidence, defined as a $50 \%$ increase of aortic diameter, was substantially reduced in Trem1-deficient animals (Supplemental Figure 2), as was lethal aorta rupture (Supplemental Figure 3), without any difference in systolic blood pressure between groups (Supplemental Figure 4). After 7 days of AngII infusion, Trem1 deficiency limited elastin degradation, as assessed by the significantly higher number of elastin layers in the abdominal aorta of AngII-infused Apoe $^{-/-}$Trem1 $^{-/-}$mice compared with the aorta of AngII-infused Apoe $\mathrm{e}^{-/-} \mathrm{Trem}^{\mathrm{I}^{+/}}$mice $(4.03 \pm 0.19$ layers vs $3.30 \pm 0.20$ layers, $P<0.05)$ (Figure $1 F)$, without any difference in local Eln mRNA levels between groups (Supplemental Figure 5). The reduction in elastin degradation in Apoe - $^{--}$ $\mathrm{Trem}^{-/-}$mice was associated with a decrease of the global MMP activity in the aortic wall, quantified using ex vivo fluorescence molecular tomography imaging (Figure 1G). Given that TREM-1 regulates cytokine production $(17,18)$, we next investigated the local immunoinflammatory response and found decreased $I l 1 b$, Tnfa, Mmp2, and Mmp9 mRNA expression in the aorta of AngIIinfused $\mathrm{Apoe}^{-/-} \mathrm{Trem1}^{-/-}$mice compared with AngII-infused control mice (Figure $1 \mathrm{H}$ ). Immunostaining revealed a huge decrease in macrophage content in the aorta of AngII-infused Apoe - $^{-}$Trem1 ${ }^{-1-}$ mice compared with AngII-infused $A p o e^{-/-}$Trem $^{+/+}$mice at day 7 (Figure 1I). We therefore analyzed early monocyte infiltration in the abdominal aorta by flow cytometry after enzymatic digestion. We found a lower number of infiltrating Ly $6 \mathrm{C}^{\text {hi }}$ classical monocytes in the aorta of AngII-infused Apoe -Trem1 $^{-/-}$mice, 3 days after AngII infusion (Figure 1J), but no difference in Ly6 $\mathrm{C}^{\text {lo }}$ nonclassical monocyte and neutrophil numbers between groups (Supplemental Figure 6). Our group previously reported that AngII infusion in $A_{p o e^{-/}}$mice promotes the mobilization of classical monocytes from the spleen to the blood, and ultimately to the aortic wall, contributing massively to the macrophage content in the aneurysmal aortic wall (3). Accordingly, at day 3, AngII-induced monocytosis was observed in Apoe $^{-/-}$Trem $^{+/+}$control mice but was abolished in Trem1-deficient mice (Figure 1K). Conversely, the number of Ly6C ${ }^{\text {hi }}$ classical monocytes in the spleen of Apoe ${ }^{-/-}$Trem1 $^{-/-}$mice (Supplemental Figure 7) was higher, suggesting impaired classical monocyte mobilization from the spleen to the blood in response to AngII infusion in the absence of TREM-1.

Pharmacological blockade of TREM-1 reduces AAA formation. Our results obtained in genetically modified mice suggest that inhibiting TREM-1 is protective against AAA formation. We aimed to confirm our finding in immunocompetent animals using a pharmacologic inhibitor of TREM-1 activation, validated in rodents and pigs, the dodecapeptide LR12 $(12,14)$. In a preliminary set of experiments, we found that LR12 treatment during 7 days markedly reduced the inflammatory profile in $\mathrm{Apoe}^{-/-}$mice, with a reduction in Il1b and Tnfa mRNA expression in the aorta of LR12-treated Apoe $^{-/-}$mice compared with Scramble-treated Apoe $e^{-/-}$mice (Figure 2A). In order to substantiate the effect of TREM-1 pharmacological blockade, AngII-infused Apoe ${ }^{-/-}$mice were treated with daily intraperitoneal injections of LR12 peptide (which was well toler- 
A

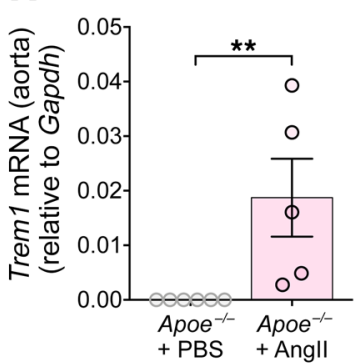

D

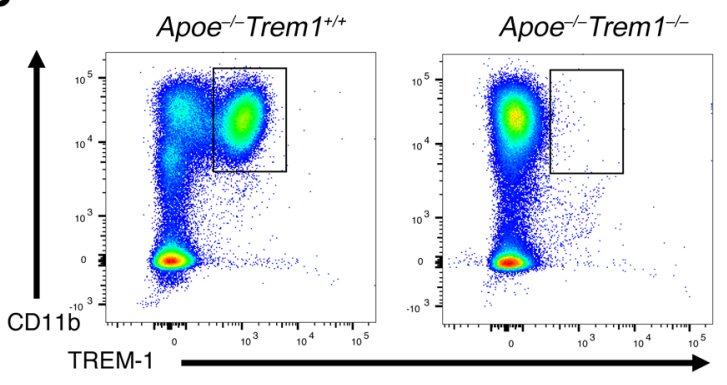

B $\mathrm{Apoe}^{-/}+\mathrm{PBS}$

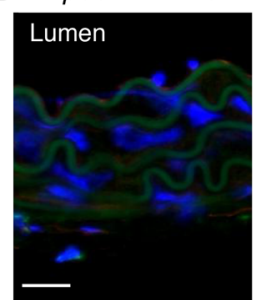

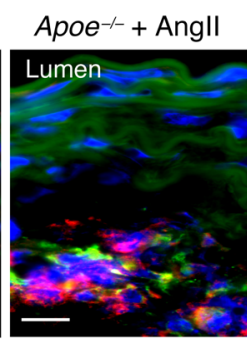

DAPI

TREM-1 CD68

\section{C}

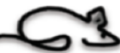

Male Apoe ${ }^{-/}$Trem1 1/+

Male Apoe ${ }^{-/}$Trem $1^{-1}$

Angiotensin II $1000 \mathrm{ng} / \mathrm{kg} / \mathrm{min}$

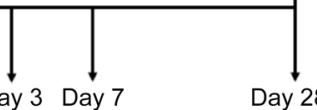

E

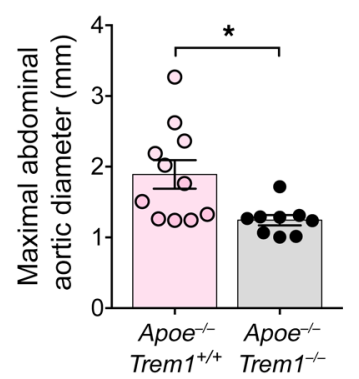

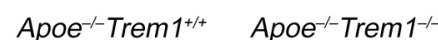

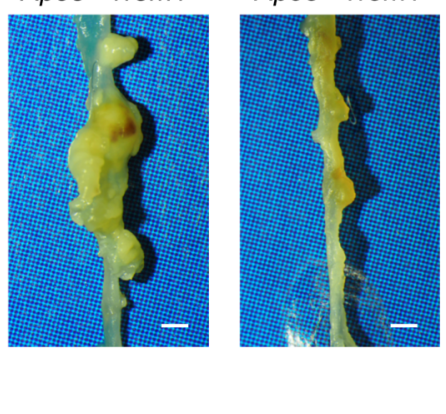

F

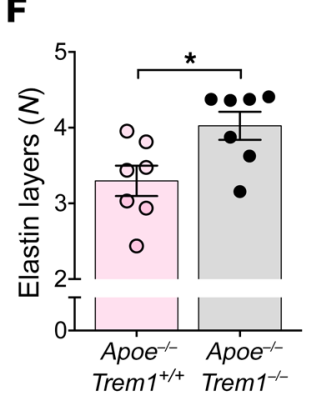

Apoe $^{-/-}$Trem $1^{+/+}$

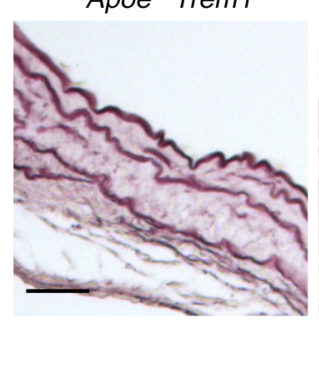

Apoe $^{-/-}$Trem1 $1^{-/-}$

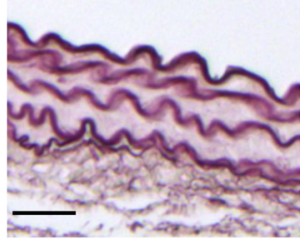



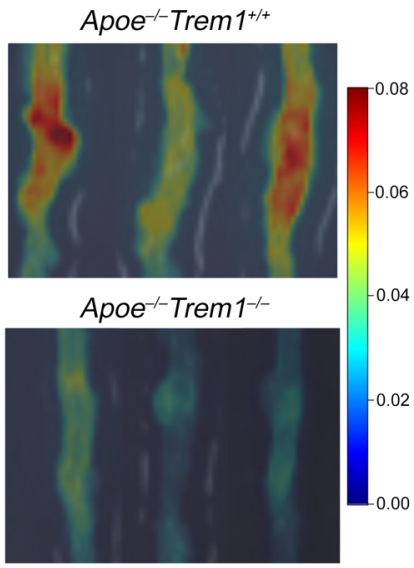

H

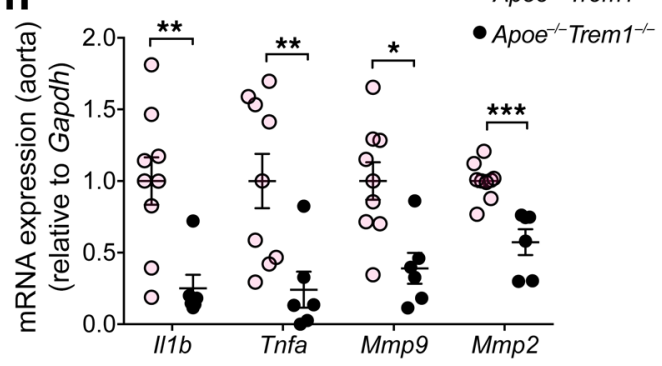

I

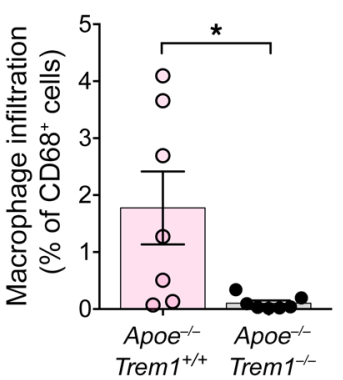

Apoe $^{-/-}$Trem $1^{+/+}$

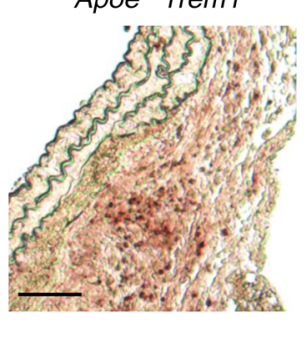

Apoe ${ }^{-/}$Trem1 1/-

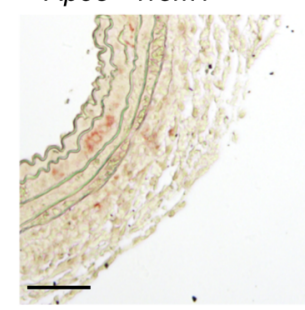

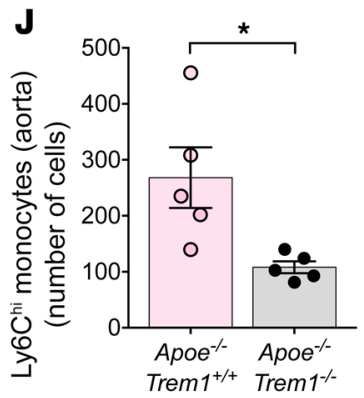
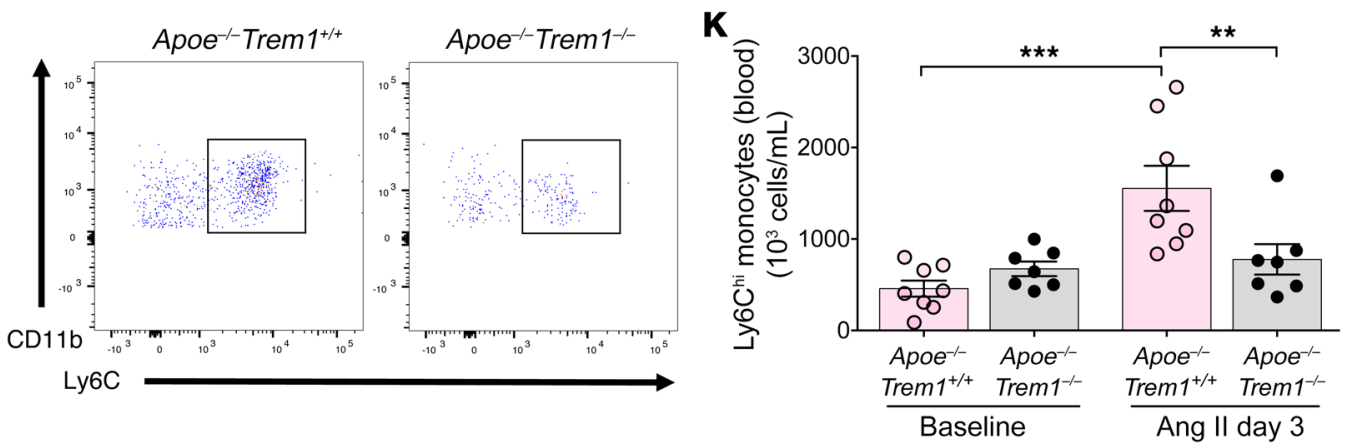
Figure 1. Trem1 genetic deletion reduces aneurysm development in angiotensin II-induced AAA. (A) Hypercholesterolemic $A p o e^{-/-}$mice were implanted with subcutaneous osmotic minipumps infusing PBS (control group) or Angll (1000 ng/kg/min). Quantification of Trem1 mRNA expression in the aorta at day 3 by RT-qPCR ( $n=6$ in PBS-infused group; $n=5$ in Angll-infused group). (B) Immunofluorescence staining in the aortic wall of PBS- (left) or Angll-infused (right) animals at day 3, DAPI (blue), TREM-1 (red), and CD68 (green). Scale bars: $50 \mu \mathrm{m}$. (C) Apoe ${ }^{-/-} \mathrm{Trem}^{+/+}$ and $A p o e^{-/-} \mathrm{Trem}^{1 /-}$ mice were implanted with subcutaneous osmotic minipumps infusing Angll (1000 ng/kg/min). Analyses were done at different time points following Angll infusion. (D) TREM-1 expression on circulating CD11b+Ly6C- monocytes of $\mathrm{Apoe}^{-/-} \mathrm{Trem}^{+/+}$(pink) and $\mathrm{Apoe}^{-/-}$ Trem 1/- mice (black) at day 3 after Angll infusion. (E) Quantitative analysis and representative photomicrographs of the maximal aortic diameter at

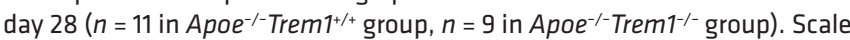
bars: $1 \mathrm{~mm}$. (F) Quantification of the number of elastin layers in the aortic wall by orcein staining at day $7(n=7 /$ group). Scale bars: $50 \mu \mathrm{m}$. (C) FMT quantification of MMP-2, $-3,-9$, and -13 activity in aorta at day 7 ( $n=4$ in Apoe $^{-/-}$Trem1 1/+ group, $n=3$ in Apoe ${ }^{-/-}$Trem $^{-/-}$group). (H) Quantification of Il1b, Tnfa, Mmp2, and Mmp9 mRNA expression in aorta at day 7 by RTqPCR ( $n=9$ in $\mathrm{Apoe}^{-/-}$Trem $^{+/+}$group, $n=6$ in $\mathrm{Apoe}^{-/-}$Trem $^{-/-}$group). (I) Quantification and representative photomicrographs of CD68+ macrophages in the aorta at day 7 ( $n=7 /$ group). Scale bars: $50 \mu \mathrm{m}$. (J) Flow cytometry quantification of Ly6 $\mathrm{Ch}^{\mathrm{hi}}$ classical monocyte infiltration in the aortic wall at day 3 ( $n=5$ in each group). (K) Flow cytometry quantification of circulating Ly6C ${ }^{\text {hi }}$ classical monocytes at day $3\left(n=8\right.$ in Apoe $^{-/-T r e m} 1^{+/+}$ group, $n=7$ in Apoe ${ }^{-/-}$Trem1 $1^{-/}$group). Kruskal-Wallis test, Mann-Whitney test; ${ }^{*} P<0.05,{ }^{* *} P<0.01,{ }^{* * *} P<0.001$.

ated) or Scramble peptide and sacrificed at day 7 (Figure 2B). We did not observe any difference in animal weight (data not shown) or in systolic blood pressure (Figure 2C) between Scramble- and LR12-treated mice. Aorta diameter was significantly lower in LR12-treated Apoe ${ }^{-/-}$mice than in Scramble-treated Apoe ${ }^{-/-}$mice $(1.19 \pm 0.08 \mathrm{~mm}$ vs $1.46 \pm 0.10 \mathrm{~mm}, P<0.05)$ (Figure 2D), as well as AAA incidence (Figure 2E). TREM-1 pharmacological inhibition reduced elastin layer degradation $(3.85 \pm 0.19$ layers in Scramble group vs $4.36 \pm 0.06$ layers in LR12 group, $P<0.05$ ) (Figure $2 \mathrm{~F}$ ) and led to a significant reduction in macrophage content in the abdominal aorta (Figure $2 \mathrm{G}$ ).

TREM-1 stimulation aggravates AngII-dependent AAA development in Apoe $e^{-/}$mice. To confirm the pathogenic role of TREM-1 in AAA development, AngII-infused Apoe $^{-/-}$mice were treated daily with an anti-TREM-1 agonistic antibody (TREM-1 Ab) or isotype control (isotype $\mathrm{Ab}$ ) for 3 or 7 days, depending on the duration of the experiment (Figure 3A). Previous studies have reported that TREM-1 Ab increases proinflammatory cytokine production by LPS-stimulated monocytes in vitro and aggravates mortality after endotoxemia (19). Here, TREM-1 stimulation in vivo aggravated the systemic proinflammatory response in AngII-infused Apoe A- $^{-1}$ mice, as shown by enhanced TNF- $\alpha$ and IL- 6 production by splenocytes from TREM-1 Ab-treated mice compared with splenocytes from isotype-treated animals (Figure 3B). Following in vivo TREM-1 Ab treatment, plasma levels of soluble TREM-1, shed after receptor activation, markedly increased (Figure 3C). Membrane TREM-1 expression on Ly6 $\mathrm{C}^{\text {hi }}$ classical monocytes was no longer detected (Supplemental Figure 8), which was likely due to receptor saturation by TREM-1 agonistic antibody.

TREM-1 stimulation aggravated AAA severity at day 28 after AngII infusion, as abdominal aortic diameters were significantly larger in TREM-1 Ab-treated Apoe ${ }^{-/}$mice as compared with isotype-treated $A p o e^{-/-}$mice $(2.1 \pm 0.7 \mathrm{~mm}$ vs $1.5 \pm 0.4 \mathrm{~mm}, P<0.05)$ (Figure 3D) and AAA incidence was significantly higher (Figure 3E), without any difference in blood pressure (Supplemental Figure 9). At day 7, TREM-1 stimulation worsened elastin degradation, as revealed by the significantly lower number of elastin layers in the abdominal aorta of AngII-infused mice treated with antiTREM-1-stimulating monoclonal antibody $(3.48 \pm 0.09$ layers in TREM-1 Ab group vs $3.85 \pm 0.03$ layers in isotype Ab group, $P<$ 0.05) (Figure $3 F$ ). At day 7, TREM-1 aggravated local proinflammatory responses, characterized by an increase in Il1b and $M m p 9$ mRNA expression in the aorta (Figure 3G). Finally, Ly6C ${ }^{\text {hi }}$ classical monocyte infiltration within the aortic wall was significantly increased in the TREM-1 Ab-treated group compared with isotype-treated mice (Figure $3 \mathrm{H}$ ), without any significant difference in neutrophil and nonclassical monocyte infiltration (Supplemental Figure 10). Ly6C ${ }^{\text {hi }}$ classical monocyte levels in the blood were not different between TREM-1 Ab-treated and isotype-treated groups (Figure 3I), suggesting that TREM-1 pathogenic activity was not restricted to the control of spleen monocyte mobilization.

TREM-1 regulates CD62L-dependent classical monocyte infiltration in the aorta. In order to understand how TREM-1 engagement could regulate classical monocyte trafficking in experimental AAA, we analyzed chemokine receptor and adhesion molecule expression on classical monocytes using mass cytometry 1 day after AngII infusion. CX3CR1 and CCR2 expression was reduced in Trem1-deficient classical monocytes in blood and spleen (Figure $4 \mathrm{~A}$ ), in agreement with our previous study showing that TREM-1 can regulate monocyte trafficking through chemokine/chemokine receptor activity after experimental acute myocardial infarction (12). Locally, we found a significant reduction of $\mathrm{Ccl}$, $\mathrm{Ccl} 3, \mathrm{Ccl} 5$, Cor2, and Cor 5 mRNA expression in the aorta of AngII-infused Apo $^{-/-}$Trem1 $^{-1-}$ mice compared with the aorta of AngII-infused Apoe $^{-/-}$Trem1 $^{+/+}$mice (Figure 4B). Conversely, TREM-1 stimulation led to a significant increase in $\mathrm{Ccl} 2, \mathrm{Ccl} 3, \mathrm{Ccl} 5, \mathrm{Ccr} 2$, and $\mathrm{Ccr} 5$ mRNA expression in the aorta of AngII-infused Apoe ${ }^{-/-}$(Figure 4C), suggesting that TREM-1 modulates monocyte trafficking partly through the regulation of chemokine/chemokine receptor activity.

We also observed that CD62L expression was markedly decreased on Trem1-deficient circulating Ly6 $\mathrm{C}^{\text {hi }}$ classical monocytes compared with control Ly6 $\mathrm{C}^{\text {hi }}$ classical monocytes, 1 day after AngII infusion (Figure 4, A and D, and Supplemental Figure 11). After 3 days of AngII infusion, we found a significant correlation between CD62L and TREM-1 expression on Ly6C ${ }^{\text {hi }}$ classical monocytes in isotype $\mathrm{Ab}$-treated Apoe ${ }^{-/-}$control mice (Supplemental Figure 12). Furthermore, TREM-1 stimulation increased the percentage of CD62L $\mathrm{L}^{+}$Ly6C ${ }^{\text {hi }}$ classical monocytes in the blood (Figure $4 \mathrm{E}$ ), as well as Sell mRNA expression in the aorta of AngII-infused Apoe ${ }^{-/-}$mice (Figure 4F). To assess whether CD62L was involved in Ly $6 \mathrm{C}^{\text {hi }}$ classical monocyte early recruitment within the aorta following TREM-1 stimulation, AngII-infused Apoe $e^{-/-}$mice receiving either stimulating TREM-1 Ab or isotype control were treated with an anti-CD62L neutralizing antibody (Figure 4G). CD62L blockade was confirmed by flow cytometry in the blood (Supplemental Figure 13). Interestingly, CD62L blockade abolished the increase of aortic classical monocyte infiltration induced by TREM-1 activation following AngII infusion (Figure 4H). 
A

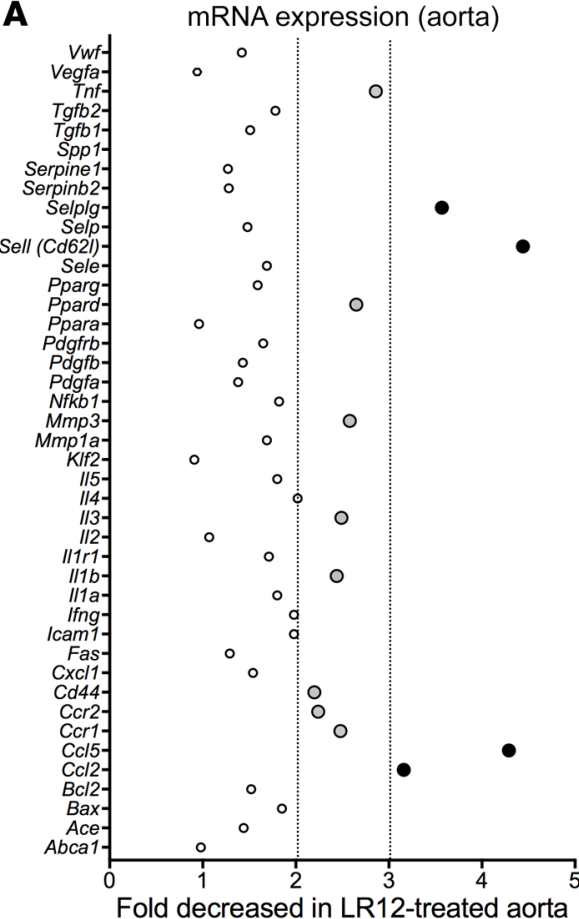

B

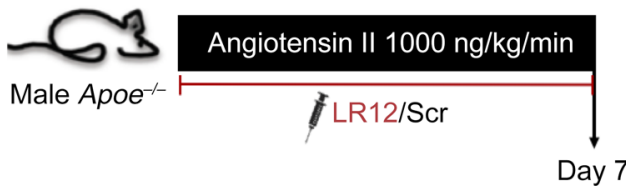

C

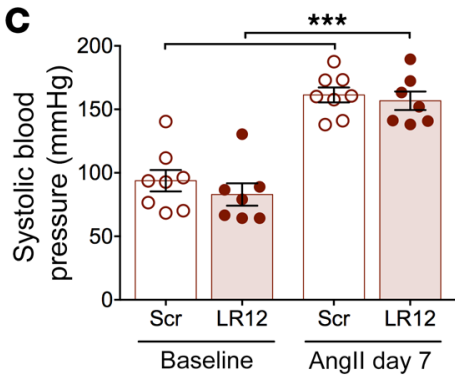

D

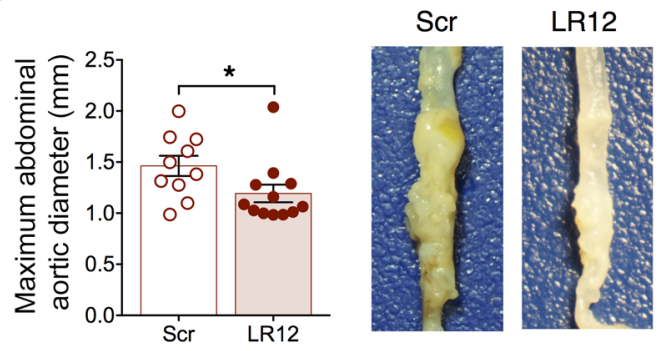

E Scr

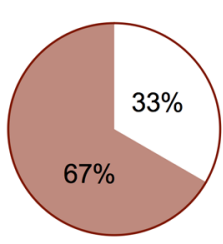

LR12

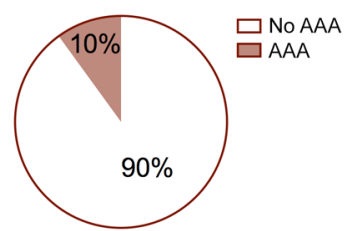

$\mathbf{F}$

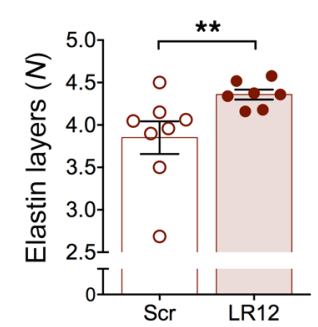

G

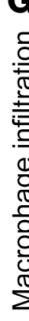

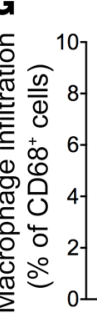

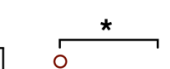

0

○
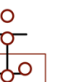

0

s
Scr

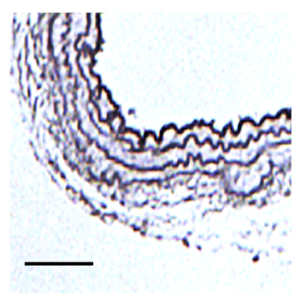

Scr

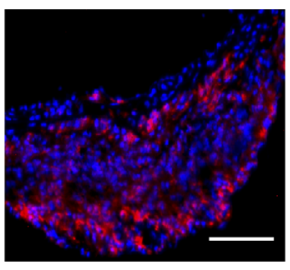

LR12

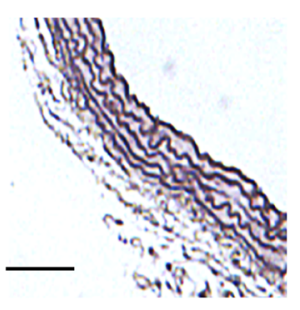

LR12

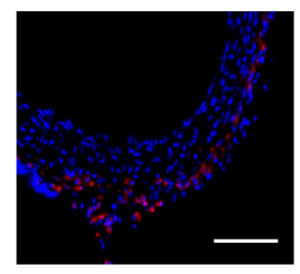

Figure 2. TREM-1 pharmacologic inhibition protects against experimental AAA. (A) Apoe $e^{-/-}$mice were treated intraperitoneally with a TREM-1 inhibitory peptide (LR12) or a control scramble peptide (Scr) $(150 \mu \mathrm{g} / \mathrm{mice} / \mathrm{d})$ for 7 days. MicroArray gene analysis was performed in the whole aorta and LR12-induced gene expression modulation was expressed as fold decreased in LR12-treated group. Light gray dots identified genes whose expression was statistically different between scramble- and LR12-treated mice with a ratio between 2 and 3 . Black dots identified genes whose expression was statistically different between scramble- and LR12-treated mice with a ratio greater than 3. (B) Apoe $e^{-/-}$mice were implanted with subcutaneous osmotic minipumps infusing Angll $(1000 \mathrm{ng} / \mathrm{kg} / \mathrm{min})$ and treated intraperitoneally with a TREM-1 inhibitory peptide (LR12) or a control scramble peptide (Scramble) (150 $\mu \mathrm{g} / \mathrm{mice} / \mathrm{d})$ for 7 days. (C) Systolic blood pressure of scramble- or LR12-treated Apoe $/$ - mice at baseline and after Angll infusion (1000 $\mathrm{ng} / \mathrm{kg} / \mathrm{min})(n=7-8 / \mathrm{group})$. (D) Quantitative analysis and representative photomicrographs of the maximal abdominal aortic diameter $(n=10$ in the scramble-treated group, $n=12$ in the LR12-treated group) and (E) AAA incidence. Scale bars: $1 \mathrm{~mm}$. (F) Histologic quantification of the number of elastin layers in the aortic wall by orcein staining. Scale bars: $100 \mu \mathrm{m}$. (G) Quantification and representative photomicrographs of CD68+ macrophages in the aorta $(n=8$ in the scramble-treated group, $n=7$ in the LR12-treated group). Scale bars: $100 \mu \mathrm{m}$. Results are displayed as the mean $\pm \mathrm{SEM}$. ${ }^{*} P<0.05,{ }^{* *} P<0.01,{ }^{* * *} P<0.001$, by Mann-Whitney test.

Angiotensin II upregulates TREM-1 expression in Ly6C ${ }^{\text {hi }}$ classical monocytes through $A T_{1} R$ engagement. Using several complementary AngII-induced experimental models, we demonstrated that TREM-1 controlled AAA development through regulation of monocyte traf- ficking. Of note, we and others have previously demonstrated that AngII is instrumental in the regulation of splenic-dependent monocyte mobilization and trafficking $(3,20)$. We therefore hypothesized that AngII might trigger TREM-1 engagement on Ly6C ${ }^{\text {hi }}$ classical 
A

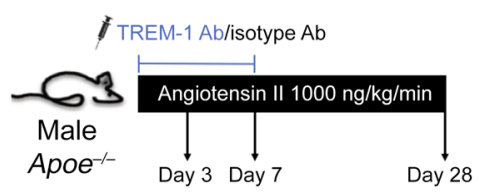

B

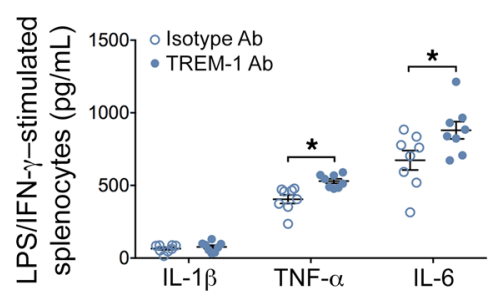

C

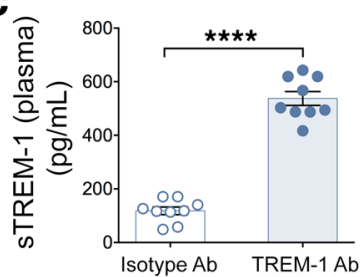

D

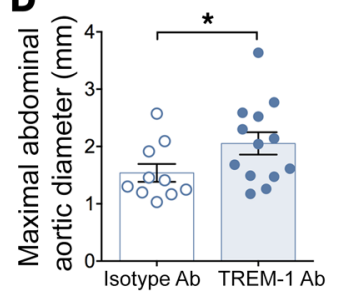

G

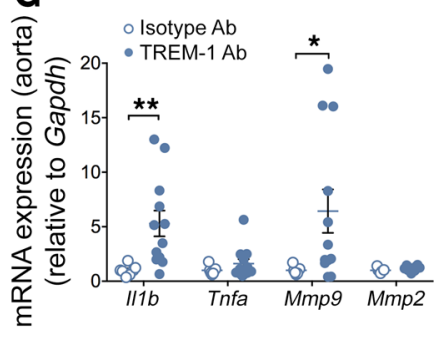

E

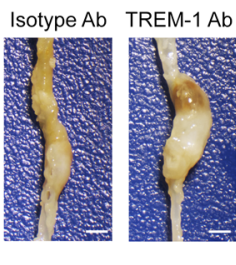

$\mathbf{F}$


I
H

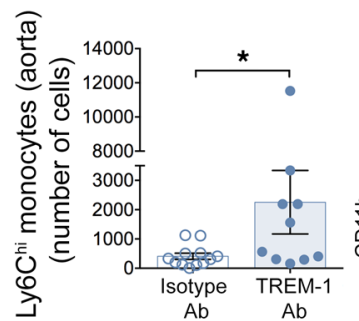

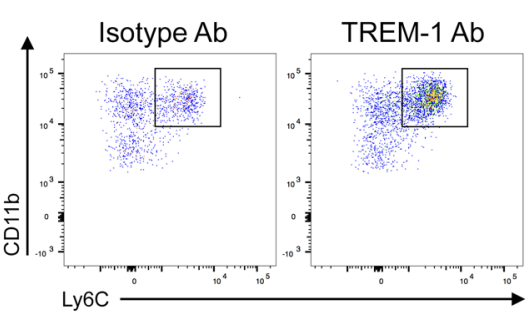

Figure 3. TREM-1 pharmacologic stimulation worsens angiotensin-II-induced AAA. (A) Apoe ${ }^{-/-}$mice were implanted with subcutaneous osmotic minipumps infusing Angll (1000 $\mathrm{ng} / \mathrm{kg} / \mathrm{min})$, and were injected intraperitoneally with an agonist TREM-1 monoclonal antibody (TREM-1 Ab) or isotype control (isotype Ab) $(200 \mu \mathrm{g} / \mathrm{kg} / \mathrm{d})$, every day for 3 or 7 days. (B) Quantification of IL-1 $\beta$, TNF- $\alpha$, and IL-6 production (ELISA) by LPS/INF- $\gamma-\mathrm{stimulated}$ splenocytes. (C) Quantification of sTREM-1 plasma levels at day 3 following Angll infusion and TREM-1 Ab or isotype Ab treatment ( $n=9 / g r o u p)$. (D) Quantitative analysis and representative photomicrographs of the maximal abdominal aortic diameter at day 28 ( $n=12$ in the isotype Ab group, $n=10$ in TREM-1 Ab group). (E) AAA incidence at day 28. Scale bars: $1 \mathrm{~mm}$. (F) Quantification and representative photomicrographs of the number of elastin layers in the aortic wall after Orcein staining at day 7 ( $n=5$ /group). Scale bars: $50 \mu \mathrm{m}$. (G) Quantification of Il1b, Tnfa, Mmp2, and Mmp9 mRNA expression in aorta at day 7 by RT-qPCR ( $n=7$ in the isotype Ab group, $n=12$ in TREM-1 Ab group). (H) Flow cytometry quantification and representative dot plots of Ly6C ${ }^{\text {hi }}$ classical monocyte number into the aortic wall and (I) in the blood ( $n=12$ in the isotype Ab group and $n=10$ in the TREM-1 Ab group). Results are displayed as the mean \pm SEM. ${ }^{*} P<0.05,{ }^{* *} P<0.01,{ }^{* * *} P<0.0001$, by Mann-Whitney test.

monocytes during AAA development. To test this hypothesis, we analyzed the myeloid expression of TREM-1 in Apoe ${ }^{-/-}$male mice infused with PBS or AngII by flow cytometry in blood and spleen at different time points (Figure 5A). At baseline, very few circulating Ly6 $\mathrm{C}^{\text {hi }}$ classical monocytes expressed TREM-1 in Apoe ${ }^{-/-}$mice, but after 3 days AngII treatment increased membrane TREM- 1 expression (Figure 5, B and C) as well as plasma levels of sTREM-1 compared with PBS (Figure 5D). Of note, we did not observe any significant change in membrane TREM-1 expression, either on circulating $\mathrm{CD}_{11} \mathrm{~b}^{+} \mathrm{Ly}_{6 \mathrm{G}^{+}}$neutrophils that almost all expressed TREM- 1 at baseline, or on Ly6 $\mathrm{C}^{\text {lo }}$ nonclassical monocytes (Supplemental Figure 14 and Supplemental Figure 15). We observed the same phenotype in nonhypercholesterolemic C57BL/6J male mice (Figure 5, E-G, and Supplemental Figure 16, Supplemental Figure 17, and Supplemental Figure 18). Such an increase in TREM-1 expression was not observed after norepinephrine infusion, despite an increase in blood pressure, suggesting that increased TREM-1 expression after AngII infusion was unlikely due to AngII-induced hypertension (Supplemental Figure 19). TREM-1 expression on Ly6 $\mathrm{C}^{\text {hi }}$ classical monocytes and sTREM-1 plasma levels increased similarly in $\mathrm{Tlr} 4^{+/+}$- and $\mathrm{Tlr} 4^{-/-}$ treated mice on day 3 after AngII infusion (Figure 5, H-J).
In order to assess the role of the 2 primary AngII receptors, $\mathrm{AT}_{1} \mathrm{R}$ and $\mathrm{AT}_{2} \mathrm{R}$, in AngII-mediated TREM-1 upregulation, we used several complementary approaches. First, we analyzed TREM-1 expression on myeloid populations in $\mathrm{AT}_{1 \mathrm{~A}} \mathrm{MUT}$ mice, a genetically mouse model characterized by $\mathrm{AT}_{1 \mathrm{~A}} \mathrm{R}$ overactivation (21). When compared with control littermate, the baseline proportion of Ly6C $\mathrm{C}^{\text {hi }}$ classical monocytes expressing TREM-1 was significantly higher in the blood of $\mathrm{AT}_{1 \mathrm{~A}}$ MUT mice (Figure 5, K and L). Next, immunocompetent C57BL/6J animals were infused with AngII and treated with PBS, Losartan (an $\mathrm{AT}_{1} \mathrm{R}$ blocker), or PD123,319 (an $\mathrm{AT}_{2} \mathrm{R}$ inhibitor) (Figure $5 \mathrm{M}$ ). At day 3, the AngII-induced increase in TREM-1+ Ly6C $\mathrm{C}^{\text {hi }}$ classical monocytes in the blood was abolished in Losartan-treated mice, but not in PD123,319-treated mice (Figure $5 \mathrm{~N}$ ). The role of $\mathrm{AT}_{1} \mathrm{R}$ in AngII-mediated induction of TREM-1 expression in classical monocytes was confirmed in Agtrla-deficient mice (Figure 5O). Altogether, these results suggest that AngII induced TREM-1 expression and activation on classical monocytes through $\mathrm{AT}_{1} \mathrm{R}$ engagement.

TREM-1 is expressed in the blood and in the aortic wall of patients with $A A A$. In order to evaluate the clinical relevance of our results, we assessed TREM-1 expression in human AAA, compared with 


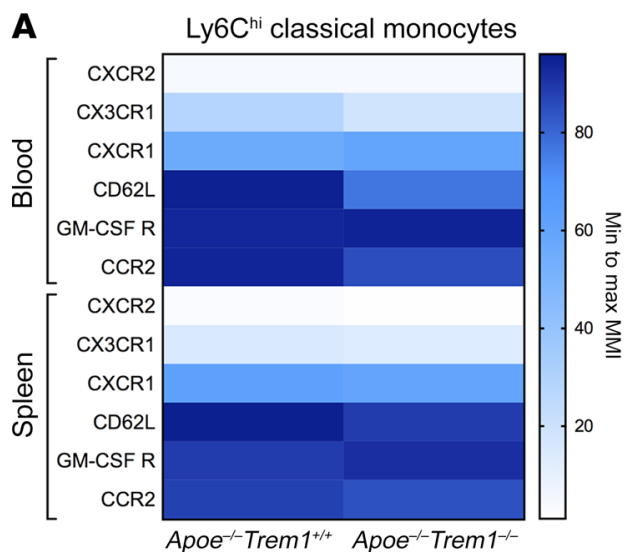

B

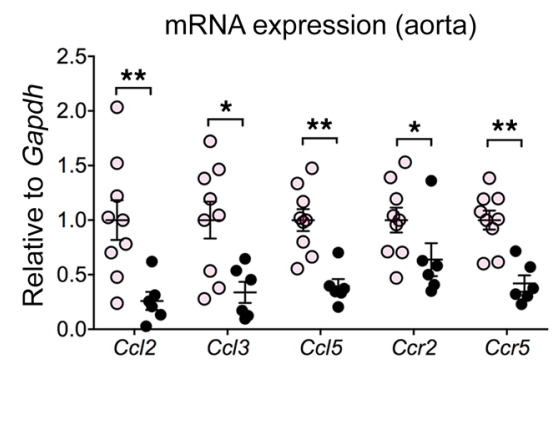

O Apoe ${ }^{-/-}$Trem $1^{+/+}$

- Apoe--Trem1-/-

E

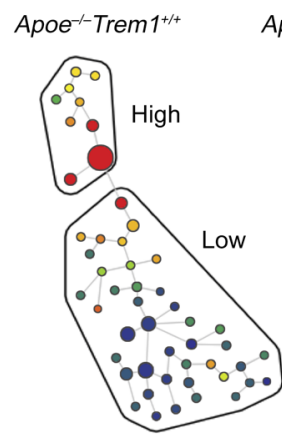

Apoe-1-Trem1 ${ }^{-1-}$
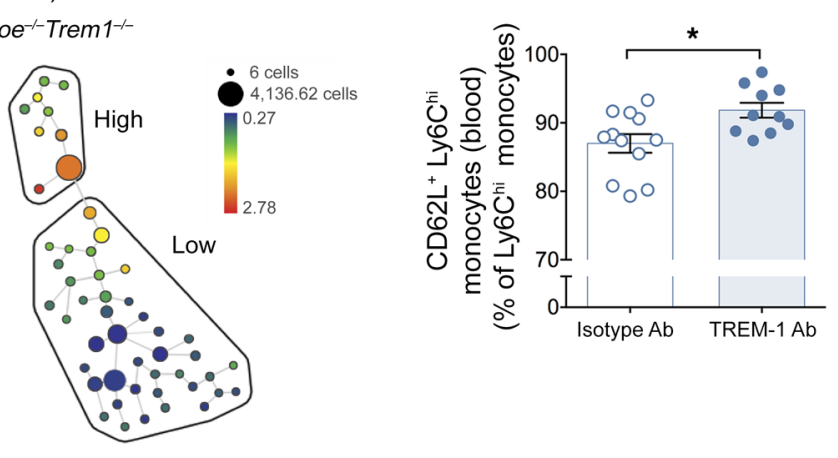

$\mathbf{F}$

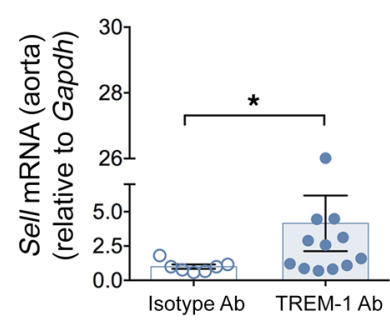

G



H

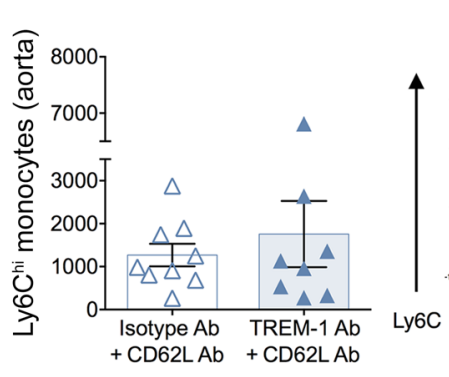

C

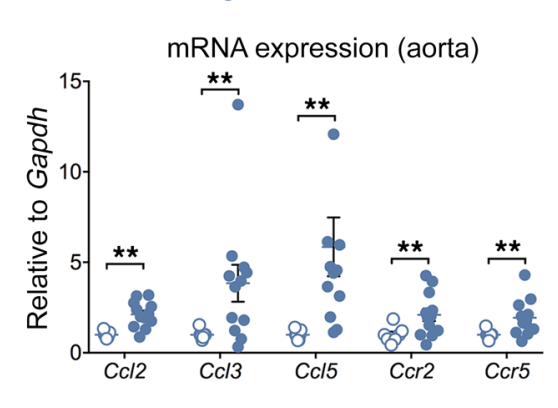

O Isotype Ab
TREM-1 Ab

Figure 4. TREM-1 modulates Ly6C ${ }^{\text {hi }}$ classical monocyte trafficking and recruitment in the aortic wall following Angll stimulation via CD62L. (A) Heatmap representation of chemokine receptors and adhesion molecule expression (expressed as mean metal intensity [MMI]), measured by mass cytometry

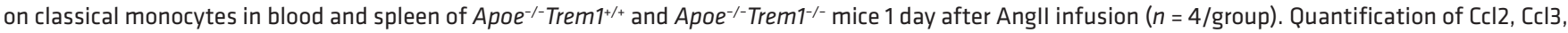

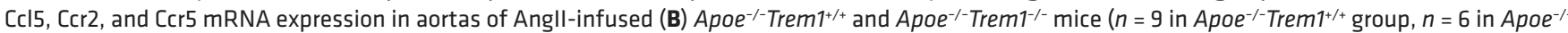
Trem $^{1 /-}$ group) and (C) isotype-treated or TREM-1 Ab-treated Apoe ${ }^{-/-}(n=7$ in isotype Ab group, $n=12$ in TREM-1 Ab group) at day 7 after Angll infusion by RT-qPCR. (D) Representative SPADE tree plots of CD62L expression on classical Ly6C hi monocytes (High) and Ly6C $\mathrm{C}^{10}$ monocytes (Low) in Apoe ${ }^{-/-T r e m} 7^{+/+}$and Apoe ${ }^{-/-}$Trem 1 1- after 1 day of Angll infusion. (E) Flow cytometry analysis of CD62L expression on Ly6C ${ }^{\text {hi }}$ classical monocytes ( $n=12$ in isotype Ab group, $n=$ 10 in TREM-1 Ab group) (F) Quantification of Sell mRNA expression by RT-qPCR in the aorta of TREM-1 Ab-treated or isotype Ab-treated Apo $e^{-/-}$mice after 3 days of Angll infusion ( $n=7$ in isotype Ab group, $n=12$ in TREM-1 Ab group). (G-H) Apoe $e^{-/-}$mice were implanted with subcutaneous osmotic minipumps releasing Angll (1000 ng/kg/min) and were treated intravenously with a neutralizing anti-CD62L antibody (CD62L Ab, 100 $\mu \mathrm{g} / \mathrm{mice} / \mathrm{d}$ ) during the first 2 days and received intraperitoneal injection of either an anti-TREM-1 Ab or an isotype Ab (control) for 3 days. (G) CD62 blockade was confirmed on Ly6C ${ }^{\text {h }}$ classical monocytes in the blood. (H) Quantification and representative dot plot of Ly6C hi classical monocyte infiltration in the aorta by flow cytometry after 3 days of Angll infusion ( $n=9$ in isotype Ab group, $n=8$ in TREM-1 Ab group). Results are displayed as the mean \pm SEM. ${ }^{*} P<0.05$, ${ }^{* *} P<0.01$, by Mann-Whitney test.

normal aortas obtained from organ donors. By quantitative PCR (RT-qPCR), we found higher levels of TREM1 mRNA expression in AAA compared with normal aortas. TREM1 mRNA expression was similar in the neck and in the dilated zone of the AAA (Figure 6A). Immunofluorescence staining showed that TREM-1 was barely detectable in normal aortas (Figure 6B), but was strongly expressed in abdominal aneurysm, its expression being mostly confined to $\mathrm{CD}^{+} 8^{+}$intimal macrophages (Figure 6, C and D). In addition, we quantified sTREM-1 levels in the plasma of healthy volunteers, patients with atrial fibrillation $(\mathrm{AF})$, and patients with AAA. Patients with AF were selected from an independent cohort and were matched $1 / 1$ with patients with AAA for cardiovascular risk factors. The characteristics of included populations are detailed in Supplemental Table 1. As expected, healthy subjects are younger 
A
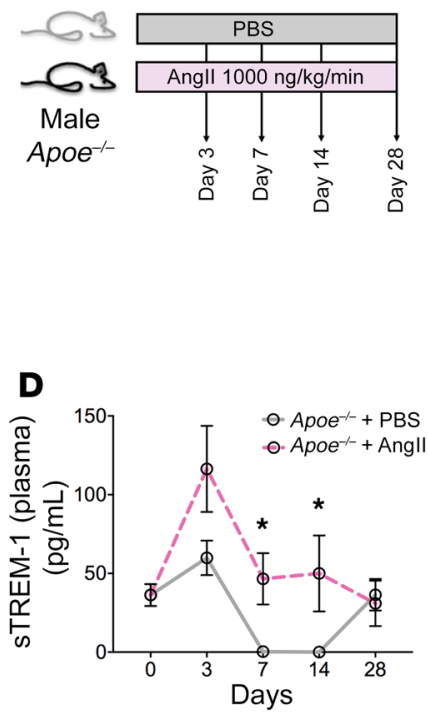

H

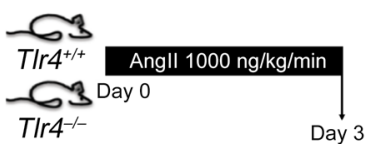

B

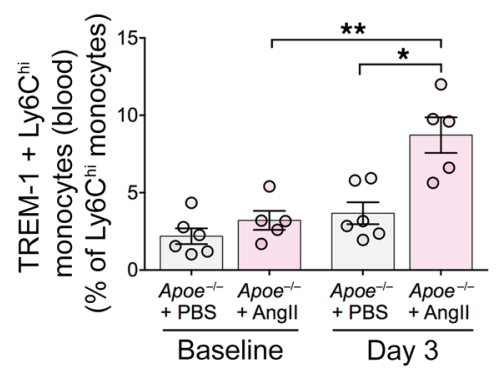

E

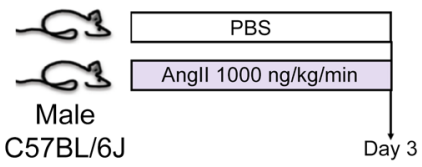

C

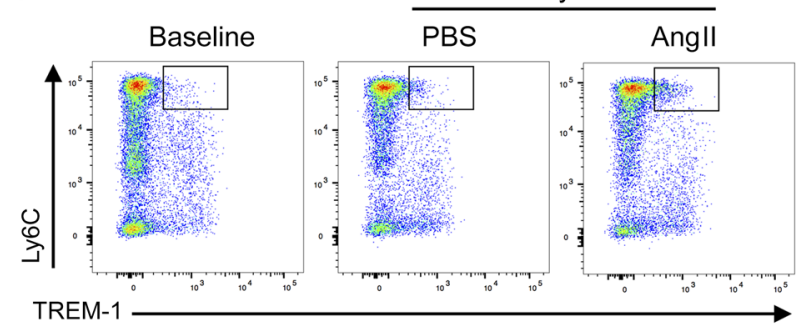

$\mathbf{F}$

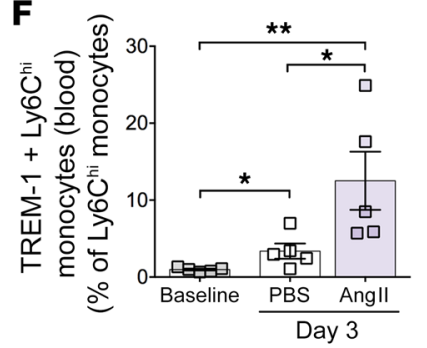

G

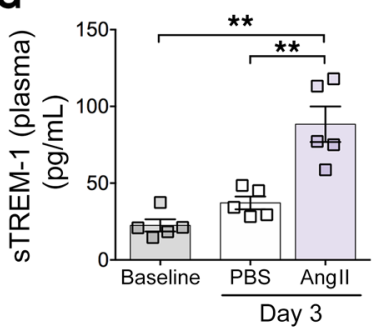

I

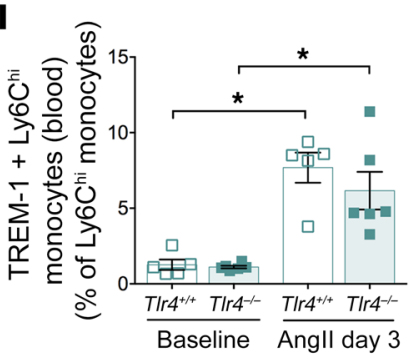

J

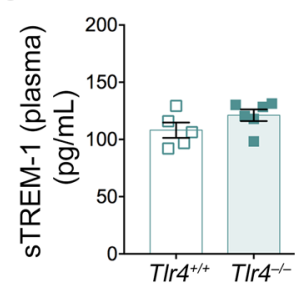

K

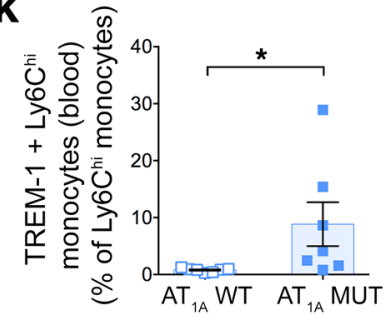

$\mathbf{L}$

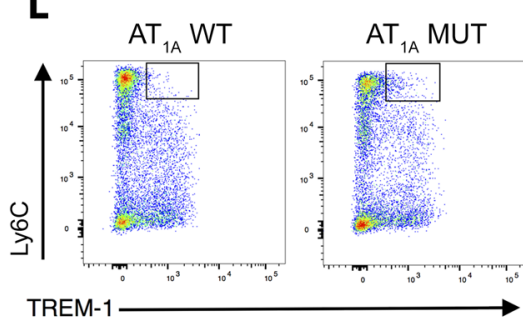

M

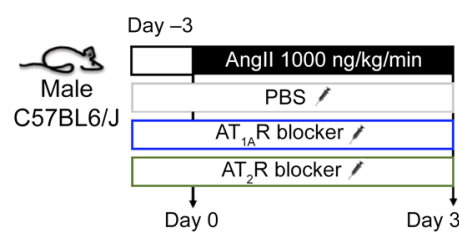

N



0

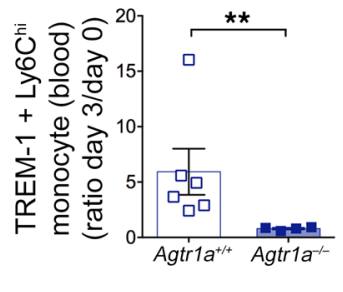

Figure 5. Angiotensin II induces TREM-1 expression on Ly6 $\mathrm{C}^{\mathrm{hi}}$ classical monocytes and sTREM-1 release through AT1R signaling, in a TLR4-independent manner. (A-D) Apoe $\mathrm{e}^{-/-}$mice were implanted with subcutaneous osmotic minipumps infusing PBS or Angll (1000 ng/kg/min). (B-C) Flow cytometry analysis and representative dot plots of TREM-1 expression on circulating Ly6C hi classical monocytes ( $n=5-6 / g r o u p)$. (D) Kinetics of plasma soluble TREM-1 following Angll infusion detected by ELISA. (E-G) C57BL/6) mice were implanted with subcutaneous osmotic minipumps infusing PBS or Angll. (F) Flow cytometry analysis of TREM-1 expression on Ly6C ${ }^{\text {hi }}$ classical monocytes in the blood and (G) quantification of plasma soluble TREM-1 by ELISA 3 days after Angll infusion ( $n=5 /$ group). (H-J) TIr $4^{+/+}$and Tlr4 ${ }^{-/-}$mice were implanted with subcutaneous osmotic minipumps infusing Angll. (I) Flow cytometry analysis of membrane TREM-1 expression on Ly6C ${ }^{\text {hi }}$ classical monocytes and (J) quantification of plasma sTREM-1 by ELISA at day 3 ( $n=5$ in TIr $4^{+/+}$group, $n=6$ in Tlr4 ${ }^{-/-}$group). (K-L) Flow cytometry analysis and representative dot plots of TREM-1 expression on circulating Ly6C ${ }^{\text {hi }}$ classical monocytes in AT1A MUT mice ( $n=7 /$ group). (M-N) C57BL/6] mice were injected intraperitoneally 3 days with PBS, AT1R blocker (Losartan), or AT2R blocker (PD123,319) prior to subcutaneous osmotic minipumps implantation infusing Angll for another 3 days. (N) Variation of TREM-1 membrane expression after 3 days of Angll infusion on Ly6C ${ }^{\text {hi }}$ classical monocytes was quantified by flow cytometry ( $n=7$ in PBS group, $n=8$ in AT1R blocker group, $n=9$ in AT2R blocker group). (0) Flow cytometry analysis of the variation of TREM-1 expression on Ly6C ${ }^{\text {hi }}$ classical monocytes after 3 days of Angll infusion in Agtr1a ${ }^{+/+}$and Agtr1a ${ }^{-/-}$mice ( $n=6$ in Agtr1a ${ }^{+/+}, n=4$ in Agtr1a ${ }^{-/-} /$group/time point). Results are displayed as the mean \pm SEM. ${ }^{*} P<0.05,{ }^{*} P<0.01$, by Mann-Whitney test (B, D, F, G, I, $\mathbf{K}$, and $\mathbf{0}$ ), and by Kruskal-Wallis test (N).

and have less frequent cardiovascular risk factors. Characteristics of patients with AF or AAA were similar except for smokers, who are more frequent in the AAA group. We found that sTREM-1 plasma levels were more than 3-fold higher in patients with $\mathrm{AF}$ (381 $\pm 238 \mathrm{pg} / \mathrm{mL})$ and in patients with AAA $(299 \pm 146 \mathrm{pg} / \mathrm{mL})$ when compared with healthy individuals $(119 \pm 86 \mathrm{pg} / \mathrm{mL} ; P<0.001)$. In addition, sTREM-1 plasma levels were significantly higher in AAA patients when compared with AF patients $(P=0.003)$ (Figure 6E).

Finally, by RT-qPCR, we found higher levels of SELL mRNA expression in AAA compared with normal aortas (Supplemental 
A

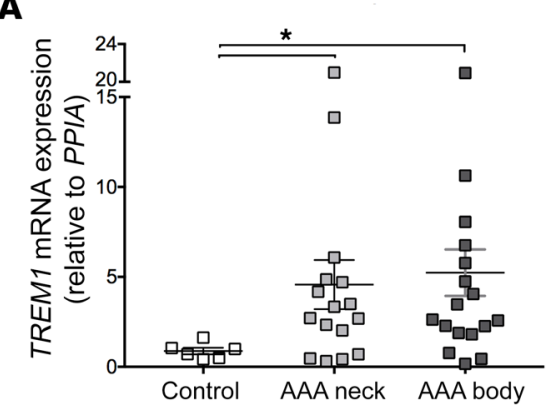

E



B
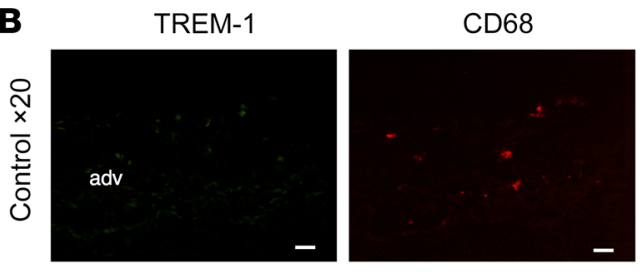

C
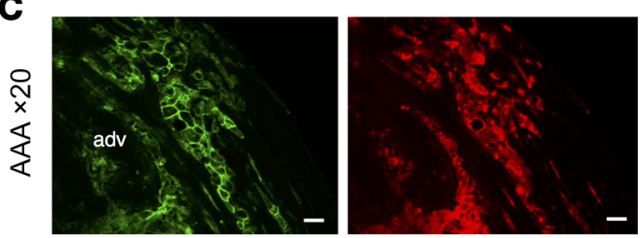

D
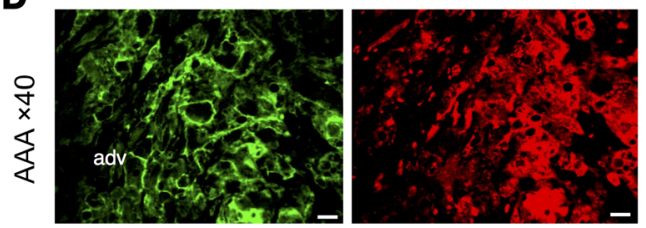

$\mathbf{F}$

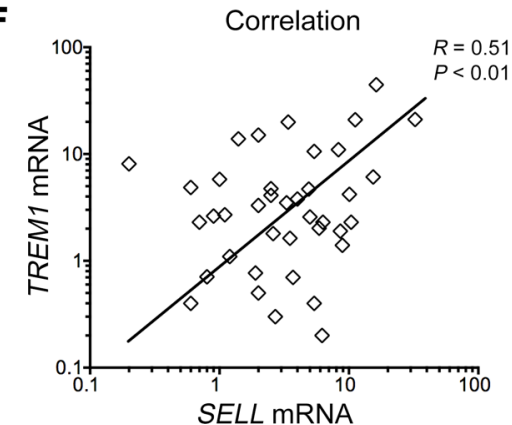

Figure 6. TREM-1 expression in human AAA. (A) TREM-1 mRNA expression was quantified in normal aorta (from organ donors) and in AAA (neck and body) by RT-qPCR. TREM-1 mRNA expression was significantly higher in AAA tissues than in normal aorta tissue. (B) Immunofluorescence staining confirmed that TREM-1 is not expressed in normal aorta (C-D) but is strongly expressed in AAA tissues and localizes with CD68 ${ }^{+}$macrophages. Scale bars: $100 \mu \mathrm{m}$. (E) Soluble TREM-1 levels were quantified by ELISA in the plasma of healthy volunteers or in the group of patients with AAA or AF. (F) Correlation between TREM1 and SELL mRNA in aorta (pooled normal and AAA). Results are displayed as the mean $\pm \mathrm{SEM}$. ${ }^{*} P<0.05,{ }^{* *} P<0.01,{ }^{* * *} P<0.001$, by Kruskal-Wallis test (A and $\mathbf{E})$ and Pearson correlation test $(\mathbf{F})$.

Figure 20), and SELL mRNA levels significantly correlated with TREM1 mRNA levels in the aorta (Figure 6F).

\section{Discussion}

In an AngII-induced mouse model, we identified a pathogenic role for TREM-1 in AAA pathophysiology. TREM-1 orchestrated classical monocyte mobilization from the spleen to the blood through chemokines/chemokine receptors modulation and regulated monocyte infiltration into the aortic wall through CD62L upregulation. In addition, we showed that AngII induced TREM-1 expression on classical monocytes through $\mathrm{AT}_{1} \mathrm{R}$ engagement, unraveling a relation between the renin-angiotensin system and the TREM-1 pathway.

Early macrophage infiltration in the aortic wall is a hallmark of AAA development both in mouse and in human $(22,23)$. Macrophages largely participate in aorta wall degradation and remodeling through the production of chemokines, cytokines, and proteases. Macrophages detected in the aneurysmal aorta are mostly derived from circulating monocytes. Our group has previously shown that AngII infusion promotes acute mobilization of classical monocytes from the spleen to the blood within a few days, and such monocytosis is mandatory for experimental AAA formation (3). Interestingly, Trem 1 deficiency abolished AngII-induced early monocytosis, and reduced AAA incidence and severity. This is in agreement with our previous finding in experimental acute myocardial ischemia, a condition characterized by acute production of endogenous AngII, showing that monocytosis occurring after coronary occlusion was prevented in Trem1 ${ }^{-/}$mice, as well as by TREM-1 pharmacological blockade (12). The mechanisms driving splenic monocyte mobilization following AngII stimulation are still in debate. We found a decrease in CCR2 and CX3CR1 expression on classical monocytes at day 1 after AngII infusion in both

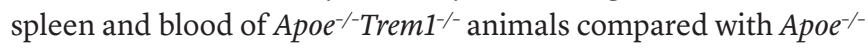
$\mathrm{Trem1}^{+/+}$control mice. The preventive effect of Trem 1 deficiency on AngII-induced monocytosis was unlikely due to decreased CCR2 expression, as previous work showed that AngII-induced monocytosis was not affected by $\mathrm{Ccr} 2$ deficiency (20). CCR5 and its ligand CCL5 are more attractive candidates to explain how TREM-1 
orchestrates AngII-induced monocyte mobilization. Mikolajczyk et al. recently reported impaired leukocyte recruitment in the aorta in Ccl5 deficient mice infused by AngII (24). In addition, pharmacological blockade of CCR5 protects against AAA development (5). In our study, Trem1 deletion or pharmacological blockade were associated with a significant reduction in Ccl5 and Ccr5 mRNA expression in the aortic wall, while antibody-mediated TREM-1 stimulation substantially increased their expression. These observations suggest that the CCL5/CCR5 axis was likely involved in the pathogenic effects of TREM-1 in AngII-induced AAA.

Cytometry by time of flight (CYTOF) characterization of classical monocytes at day 1 after AngII infusion revealed a substantial reduction in CD62L expression in Trem1-deficient cells in the spleen and in the blood. Following AngII, TREM-1 expression correlated with CD62L expression on classical monocytes. Moreover, antibody-mediated TREM-1 stimulation increased CD62L expression on circulating Ly6 $\mathrm{C}^{\text {hi }}$ classical monocytes and Sell mRNA levels in the aorta after 3 days of AngII infusion, suggesting that TREM-1 regulated AngII-induced CD62L expression. CD62L, a member of selectin family expressed by leukocytes, plays a pivotal role in endothelial adhesion. CD62L is also involved in AAA pathophysiology, as CD62L-deficient $\mathrm{Sell}^{-/} \mathrm{Apoe}^{-/-}$mice were protected against AAA development. In our study, we found that CD62L blockade abolished the early increase in monocyte infiltration induced by TREM-1 stimulation, suggesting that TREM1 orchestrated monocyte adhesion through the regulation of CD62L expression. We detected SELL expression in human AAA by qPCR, and SELL mRNA levels substantially correlated with TREM1 mRNA levels. In humans, a marked association between a TREM1 gene polymorphism and CD62L plasma levels has been reported, supporting a pathophysiological link between TREM-1 and CD62L pathways in human AAA disease (25).

The protective effect of TREM- 1 blockade might be due to local modulation of vascular inflammation. Il1b and Tnfa mRNA expression was reduced in the aorta of Trem1-deficient mice, and $I l 1 b$ mRNA expression was increased by TREM-1 stimulation. Both cytokines have been shown to promote experimental AAA $(26,27)$ through vascular cell activation and immune cell recruitment. Trem1 deficiency also reduced MMP-2, -3, -9, and -13 activities. MMPs have been clearly identified as major contributors in AAA pathophysiology. Levels of active MMP-2 and MMP-9 are substantially increased in human AAA tissue samples (28). In mouse models of AAA, MMP-2 and MMP-9 activities in the aortic wall increase over time (29). In the periaortic $\mathrm{CaCl}_{2}$ injury model of AAA, lesion formation was fully blocked in both Mmp9- and Mmp2-deficient mice (30). MMP-12 is also expressed in mouse (31) and human (32) aortic aneurysmal tissues, mainly by aortic macrophages. While MMP-9 is induced after cell activation, MMP-12 is constitutively expressed by macrophages, and its expression may further be modulated by several stimuli $(33,34)$. Our group has previously reported that gene deletion of $\mathrm{Mmp12}$ reduced AAA severity in the AngII/anti-TGF- $\beta$ mouse model (31).

Decreased $I l 1 b$ and Tnfa mRNA expression, as well as decreased metalloprotease activity in the aorta of AngII-infused Apoe -/-Trem1 $^{-/-}$mice, likely resulted from reduced macrophage content in the aorta. This might also be partly due to the switch of Trem1-deficient macrophage phenotype toward a less inflam- matory profile. Indeed, TREM-1 inhibition has been shown to promote an antiinflammatory phenotype in both human monocytes and mouse macrophages after LPS (35) or oxidized LDL stimulation (36) in vitro. Also, in a model of ureteral obstruction-induced nephritis, Trem 1 deficiency reduced M1 macrophage polarization within the kidney, as illustrated by decreased Nos 2 and increased Arg1 mRNA expression (37).

Vascular protection associated with TREM-1 invalidation/ blockade was unlikely mediated by neutrophil modulation. TREM-1 inhibition had no effect on neutrophil mobilization following AngII infusion, nor on neutrophil infiltration in the aortic wall.

TREM-1 is constitutively expressed on neutrophils and nonclassical monocytes (8). In our study, we showed that AngII infusion increased TREM-1 expression specifically on classical monocytes. Using genetically modified mice as gain- and loss-offunction models ( $\mathrm{AT}_{1 \mathrm{~A}}$ MUT and Agtrla $^{-/-}$mice, respectively) and pharmacological agents ( $\mathrm{AT}_{1} \mathrm{R}$ and $\mathrm{AT}_{2} \mathrm{R}$ blockers), we found that $\mathrm{AT}_{1} \mathrm{R}$ receptor engagement was instrumental in TREM-1 upregulation, whereas $\mathrm{AT}_{2} \mathrm{R}$ was not involved. We did not specifically show that AngII directly activated $\mathrm{AT}_{1} \mathrm{R}$ receptor on monocytes to promote TREM-1 expression, but both $\mathrm{AT}_{1} \mathrm{R}$ receptor expression on monocytes and AngII-mediated activation have been previously reported (20). TLR4 stimulation is a major trigger of TREM-1 expression and engagement, and TLR4 has been demonstrated to mediate AngII-induced vascular remodeling (38). Here, we found that AngII-induced TREM-1 expression on Ly6 $\mathrm{C}^{\text {hi }}$ classical monocytes in a TLR4-independent manner, since TREM-1 upregulation after AngII stimulation was still observed in Tlr4 ${ }^{-/-}$ mice. Following activation, membrane-bound TREM-1 is shed by metalloproteases and released in the milieu. Plasma sTREM-1 has been shown to be increased in sepsis (19) and in acute myocardial infarction, both in mice and human (12). In our study, we observed that baseline sTREM-1 was barely detected in the plasma of C57BL/6J and Apoe $e^{-/}$mice but was markedly increased following AngII infusion and remained elevated for 14 days, suggesting prolonged TREM-1 activation.

Despite the central role of the immune response in the development of AAA (1), most of the therapeutic strategies targeting innate immune pathways have failed so far (39); a few are still being tested in clinical trials (40). We believe that a TREM-1 inhibition-based multitarget approach, as obtained with the inhibitory LR12 dodecapeptide, might provide extended benefit and will be worthy of clinical testing. The relevance of such an approach is further supported by our clinical findings of increased levels of TREM1 mRNA expression in human aortic aneurysms and the significant increase in sTREM-1 plasma levels in AAA patients. The safety, tolerability, and pharmacokinetics of TREM-1 blockade using LR-12 peptide Nangibotide in healthy volunteers have been validated (41) and a proof-of-concept therapeutic trial is currently ongoing in patients with sepsis (ClinicalTrials.gov identifier: NCT04055909) (42). We speculated that patients with small AAA represent the population of choice that could benefit from LR-12 treatment to limit AAA growth, because TREM-1 inhibition would prevent continuous myeloid cell recruitment into the aortic wall, sustained pathogenic MMP activity, and vascular inflammation.

In conclusion, we identify TREM-1 as a critical contributor to AAA pathophysiology through the regulation of both monocyte 
trafficking and vascular wall inflammation. Targeting TREM-1 could represent a novel potential therapeutic approach to limit AAA expansion in human that should be evaluated in the future.

\section{Methods}

Human samples analysis. Human samples were obtained from patients with AAA and from organ donors at the University Hospital of Nice (Nice, France). This study involves a previously published clinical cohort $(43,44)$. The diagnosis of AAA was defined as a dilatation of the abdominal aorta with a diameter greater than $30 \mathrm{~mm}$ and was checked by a vascular surgeon based on CT scans. Blood samples were obtained after a peripheral vein puncture after 8 to 12 hours fasting. They were collected in $5 \mathrm{~mL}$ BD Vacutainer tubes coated with EDTA. Samples were centrifuged at $20^{\circ} \mathrm{C}$ for 10 minutes at $3000 \mathrm{~g}$ and aliquots of plasma were stored at $-80^{\circ} \mathrm{C}$ until analysis. Infrarenal abdominal aortic tissue was harvested from patients undergoing elective open AAA repair. Healthy abdominal aortic tissue was obtained from patients in a state of brain death. Tissues were immediately snap frozen in liquid nitrogen and stored at $-80^{\circ} \mathrm{C}$. Plasma levels of TREM- 1 were also measured in a cohort of patients with atrial fibrillation having the same CV risk factors than patients with AAA (Saint-Antoine Hospital, AP-HP, Paris, France; FASTRHAC cohort, ID RCB: 2014-A00280-47; Clinical Trial.gov: NCT02741349).

RNA extraction and analysis. RNA from tissue samples was extracted using Trizol (Life Technologies). For qPCR, RNA was reverse transcribed using Quantitec Reverse Transcription Kit (Qiagen). Reverse-transcribed cDNAs were quantified by TB Green Premix Ex Taq-based (Tli RNase H Plus, TaKaRa Bio) qPCR using specific oligonucleotides on a StepOnePlus Real-Time PCR apparatus (Thermo Fisher Scientific). The relative amount of all mRNA was calculated using the comparative $\Delta \Delta \mathrm{Ct}$ method using peptidylprolyl isomerase A (PPIA), coding for cyclophilinA as housekeeping gene. The following primer sequences were used: TREM1: forward 5'-GCC TTG TGC CCA CTC TAT ACC A-3', reverse 5'-TGG AGA CAT CGG CAG TTG AC-3'; SELL: forward 5'-TTA CCT CTG CAT GTA CCT TCA TCT G-3', reverse 5'-CAC CCT CCT TAA TCA TTG AGA AAC TT-3'; PPIA A: forward 5'-GCA TAC GGG TCC TGG CAT CTT GTC C-3', reverse 5'-ATG GTG ATC TTC TTG CTG GTC TTG C-3'.

Soluble TREM-1 levels. TREM-1 plasma concentration was measured using Quantikine ELISA Human TREM-1 Immunoassay (DTRM10C, R\&D Systems). The assay was performed according to the manufacturer's protocol.

Immunofluorescence staining. The specimens were fixed in formalin and embedded in paraffin (FFPE). Double immunofluorescence was performed as follows. The FFPE sections were first incubated overnight with a TREM-1 goat polyclonal antibody (AF1278, R\&D Systems) at 1:50 dilution (45) and revealed with an anti-goat biotinylated secondary antibody at 1:500 (BA-9500, Vector Laboratories), followed by a streptavidin-Alexa Fluor 488 conjugate at 1:500 (S32354, Thermo Fisher Scientific). After rinsing, the sections were incubated with a second primary monoclonal mouse anti-CD68 antibody at 1:100 (M081401-2, clone KP1, Agilent Technologies) to characterize macrophages. Sections were revealed by an anti-mouse antibody conjugated to Alexa Fluor 594 at 1:500 (A-21203, Thermo Fisher Scientific). Negative controls were obtained by omitting one of the primary antibodies.

Animal studies. Apoe - $^{-/}$Trem1 $^{-/-}$mice (null for both Apoe and Trem1 genes) have been described previously (45). $\mathrm{AT}_{1 \mathrm{~A}} \mathrm{MUT}$ mice, exhibit- ing a constant activation of angiotensin II receptor type 1A signaling, were provided by E. Clauser (Paris Research Cardiovascular Center, Inserm U970, Paris, France) (21). Agtr1 $a^{-/-}$mice have been previously described (46). Tlr $^{-/-}$mice were a gift of S. Akira (Department of Host Defense, Research Institute for Microbial Diseases, Osaka University, Osaka, Japan) and were provided by F. Pène (Institut Cochin, Inserm U1016, CNRS UMR8104, Université de Paris, Paris).

Angiotensin II infusion. Angiotensin II (Sigma-Aldrich) was infused in 8- to 10-week old male mice via subcutaneous osmotic pumps (Alzet) releasing a constant concentration of $1000 \mathrm{ng} / \mathrm{kg} / \mathrm{min}$ for a maximum of 28 days. Maximum abdominal aortic diameters were assessed by measuring external aortic diameter from images taken at the end of the experiment. AAA incidence was determined using a threshold $(1.38 \mathrm{~mm})$ corresponding to a $50 \%$ increase of the mean maximum abdominal aortic diameter of a pool of normal Apoe $e^{-/}$mice.

Norepinephrine infusion. Norepinephrine (Sigma-Aldrich) in $0.2 \%$ ascorbic acid was infused in 8- to 10-week old male mice via subcutaneous osmotic pumps (Alzet, Charles River Laboratories) releasing a constant concentration of $5.6 \mathrm{mg} / \mathrm{kg} / \mathrm{j}$ for 3 days.

$A T_{1} R$ and $A T_{2} R$ blockade. C57BL/6J mice (Janvier Labs) were injected intraperitoneally with $\mathrm{PBS}, \mathrm{AT}_{1 \mathrm{~A}} \mathrm{R}$ blocker (Losartan potassi-

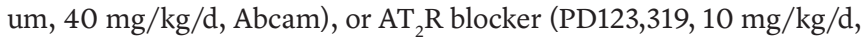
Sigma-Aldrich) 3 days before pump implantation and until the end of the experiment. Blood was harvested just before pump implantation and after 3 days of AngII infusion.

Anti-TREM-1 monoclonal antibody. Apoe ${ }^{-/-}$mice were infused with AngII (1000 ng/kg/min) for 3, 7, or 28 days. Animals were blindly randomized to receive a monoclonal anti-TREM-1 agonistic antibody (TREM-1 Ab, 0.2 mg/kg/d, MAB1187, clone 174031, R\&D Systems) or an IgG isotype control antibody (isotype $\mathrm{Ab}, 0.2 \mathrm{mg} / \mathrm{kg} / \mathrm{d}, \mathrm{MAB006}$, clone $54447, \mathrm{R} \& \mathrm{D}$ Systems) intraperitoneally once a day for 3,7 , or 5 days, respectively. These experiments were repeated several times in order to perform different tissue analyses.

Anti-CD62L monoclonal antibody. A functional grade neutralizing purified anti-mouse CD62L antibody (CD62L Ab, $0.1 \mathrm{mg} / \mathrm{kg} / \mathrm{d}$, 16-0621-86, clone MEL-14, eBioscience) was intravenously injected on day 0 and day 1 in mice infused with AngII and subjected to TREM-1 $\mathrm{Ab}$ or isotype $\mathrm{Ab}$ treatment for 3 days (47).

TREM-1 inhibitory peptide LR-12. LR12 (LR12, LQEEDAGEYGCM) and LR12-scramble (Scr, the inactive control peptide containing the same amino acids but in a randomized sequence order) were chemically synthesized (Pepscan Presto BV) as $\mathrm{COOH}$ terminally amidated peptides. The correct peptides were obtained with greater than $99 \%$ yields and were homogeneous after preparative purification, as confirmed by mass spectrometry and analytic reversed-phase high-performance liquid chromatography. These peptides were free of endotoxin. Animals were blindly randomized to receive $150 \mu \mathrm{g}$ per mouse of LR12 or LR12-scramble peptides intraperitoneally once a day for 7 days. This dose was shown to inhibit TREM-1 pathway activation in studies on septic shock $(14,15)$ and acute myocardial infarction (12).

Characterization of aneurysmal lesions. The suprarenal region of the abdominal aorta, subjected to AAA development, was harvested and embedded in both cryostat and paraffin and 7- $\mu$ m thick sections were used for histology and immunohistochemistry analysis. Sections were stained with Orcein (MMFrance) for detection of elastin layers. The mean number of elastin layers was quantified by a researcher blinded to the experimental protocol (4 measurements/section, $8 \mathrm{sec}$ - 
tions/mouse). Sections were stained for macrophages using a rat antimouse CD68 primary antibody (MCA1957, clone FA-11, AbD Serotec) revealed with an anti-rat Cy5 secondary antibody (712-175-153, Jackson ImmunoResearch). The percentage of cellular area positive for CD68 staining (macrophages) was quantified using Histolab software (Microvision) and the $\mathrm{CD} 8^{+}$area/total aortic wall area (media+ adventitia) ratio was calculated. TREM-1 immunostaining and colocalization with macrophages were detected using a polyclonal goat anti-mouse TREM-1 antibody (AF1187, R\&D Systems), revealed with an anti-goat Cy5 secondary antibody (705-175-147, Jackson ImmunoResearch), and rat anti-mouse CD68 antibody revealed with an anti-rat AF188 secondary antibody (712-545-153, Jackson ImmunoResearch). In each experiment, histology quantifications were done by a researcher blinded to the experimental protocol.

Splenocytes culture and quantitation of cytokine release. Splenocytes were cultured in RPMI 1640 medium supplemented with Glutamax (Thermo Fisher Scientific), 10\% FCS, and antibiotics. For cytokine measurements, 1 million splenocytes were stimulated with LPS (10 $\mu \mathrm{g} / \mathrm{mL})$, with or without IFN- $\gamma(100 \mathrm{IU} / \mathrm{mL})$ for 24 hours. IL-10, IL-1 $\beta$, IL-6, and TNF- $\alpha$ production in the supernatants was measured using specific ELISAs (R\&D Systems, BD Biosciences).

Quantitation of plasmatic STREM-1 release. Mouse plasma was obtained by centrifugation of heparinized blood collected from the retroorbital sinus. The concentration of sTREM- 1 was determined using a commercially available ELISA (DuoSet; R\&D Systems), according to the manufacturer's instructions.

Fluorescence molecular tomography (FMT) imaging. Twenty-four hours before sacrifice, AngII-infused animals were anesthetized with isoflurane and received $150 \mu \mathrm{L}$ intravenously of a fluorescent imaging probe MMPsense 680 (NEV 10126, PerkinElmer). The probe is optically silent in its unactivated state and becomes highly fluorescent following activation by MMPs including MMP-2, -3, -9, and -13. Images were acquired using a fluorescence molecular imaging system (FMT 2500TM, VisEnMedical).

Flow cytometry. Animals were perfused with cold PBS and whole aortas were rapidly cleaned and harvested under a dissection microscope. Aortas were minced and incubated for 30 minutes at $37^{\circ} \mathrm{C}$ in an enzymatic digestion cocktail (collagenase I, $450 \mathrm{U} / \mathrm{mL}$, SigmaAldrich), collagenase XI (125 U/mL, Sigma-Aldrich), hyaluronidase (60 U/mL, Sigma-Aldrich), DNAse (60 U/mL, Sigma-Aldrich), and elastase (0.372 U/mL, Sigma-Aldrich). Leukocyte suspension was obtained from spleen after meshing, and blood, spleen, and aorta extracellular antigens were stained with fluorescence-labeled antimouse antibodies (see below) for 30 minutes at $4^{\circ} \mathrm{C}$. Flow cytometric acquisitions were done on a BD LSRFortessa (BD Biosciences) and data were analyzed using FlowJo Software (TreeStar). Forward scatter (FSC) and side scatter (SSC) were used to gate the live cells, red blood cells, debris, cell aggregates, and doublets being excluded. In the blood and in the spleen, neutrophils were identified as $\mathrm{CD}_{11 \mathrm{~b}^{+}} /$ $\mathrm{Ly}_{6 \mathrm{G}^{+}}$and monocytes as $\mathrm{CD}_{11 \mathrm{~b}^{+} / \mathrm{Ly} 6 \mathrm{G}^{-} \text {, Ly6C }}{ }^{\text {hi }}$ or Ly6 $\mathrm{C}^{\mathrm{lo}}$. In the aorta, Ly6C ${ }^{\text {hi }}$ classical monocytes were identified as $\mathrm{CD}^{+} 5^{+} / \mathrm{CD} 11 \mathrm{~b}^{+} /$

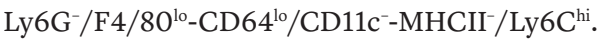

The following primary conjugated antibodies were used for the staining in the aorta: anti-CD45 (Alexa Fluor 700, clone 30-F11, BD Biosciences), anti-CD11b (BV605, clone M1/70, BD Biosciences), antiLy6C (APC-Cy7, clone AL-21, BD Biosciences), anti-Ly6G (BUV395, clone 1A8, BD Biosciences), anti-CD64 (BV421, clone X54-5/7.1,
Biolegend), anti-F4/80 (PE, clone CI:A3-1, Abcam), anti-CD11c (PerCP-Cy5.5, clone HL3, BD Biosciences), anti-MHC-II (BV510, clone M5/114.115.2, BD Biosciences), anti-TREM-1 (APC, clone 174031, R\&D Systems), anti-CD3 (PE-Cy7, clone 145-2C11, BD Biosciences), and anti-CD45R (B220) (BUV496, clone RA3-6B2, BD Biosciences).

The following primary conjugated antibodies were used for the staining in the blood and in the spleen: anti-CD11b (PE-Cy7, clone M1/70, eBioscience), anti-Ly6C (FITC, clone AL-21, BD Biosciences), anti-Ly6G (PE, clone 1A8, BD Biosciences), anti-TREM-1 (APC, clone 174031, R\&D Systems), anti-CD62L (eFluor 450, clone MEL-14, eBioscience).

Quantitative real-time PCR. Quantitative real-time PCR was performed using a Step-one Plus (Applied Biosystems). Gapdh cycle threshold was used to normalize gene expression: forward 5'-CGTCCCGTAGACAAAATGGTGAA-3'; reverse 5'-GCCGTGAGTGGAGTCATACTGGAACA-3'. The following primer sequences were used: Trem1: forward 5'-TGCGGTTGTTTCCTCTCCTGGTCTTG-3', reverse 5'-TGTGAAATAGACACCGCTGAAGGTCACT-3'; Illb:forward5'-GAAGAGCCCATCCTCTGTGA-3'; reverse 5'-GGGTGTGCCGTCTTTCATTA-3'; Tnfa: forward 5'-GATGGGGGGCTTCCAGAACT-3', reverse: 5'-CGT GGG CTA CAG GCT TGT CAC-3'; Mmp2: forward 5'-CCGAGA CCGCTATGTCCACTGT-3', reverse 5'-CCGGTCATCATCGTAGTTGGTTGT-3'; Mmp9: forward 5'-CCGTCATTCGCGTGGATAAGGAGT-3', reverse 5'-GTAGCCCACGTC CACCTGGTT-3'; Ccl2: forward 5'-CCCCACTCACCTGCTGCTA-3', reverse 5'-TACGGGTCAACTTCACATTCAAA-3'; Ccl3: forward 5'-CACTGCCCTTGCTGTTCTTCTCTGT-3', reverse 5'-ATCTGCCGGTTTCTCTTAGTCAGGA-3'; Ccl5: forward 5'-CCCCCGCACCTGCCTCACCAT-3', reverse 5'-TTGGCACACACTTGGCGGTTCCTT-3'; Ccr2: forward 5'-AAGAGGGCATTGGATTCACCACAT-3', reverse 5'-ATGCCGTGGATGAACTGAGGTA-3'; Ccr5: forward 5'-GGTGAGACATCCGTTCCCCCTACAA-3', reverse 5'-CAGGAGCTGAGCCGCAATTTGTTTC-3'; Sell: forward 5'-CGCATTCTCGGGGCTGGCATTTCT-3', reverse 5'-CTCTAATGTGGGAGATGCCTGCGTGTAA-3'. Relative expression was calculated using the $\Delta \Delta \mathrm{Ct}$ method. For the microarray gene analysis, total RNAs were retrotranscribed with RT2 First Strand Kit (SABiosciences, Tebu-bio) for PCR arrays (Mouse Innate Immune Profiler PCR Arrays; SABiosciences). All PCRs were performed in a MyiQ Thermal Cycler and quantified by iQ5 software (Qiagen). PCR array results were analyzed using PCR Array Data Analysis Software (SABiosciences) and normalized with 5 house- keeping genes (GEO accession GSE161216).

Mass cytometry (CYTOF). Cells from spleen and blood were resuspended at $1 \times 10^{7}$ cells per $\mathrm{mL}$ and stained with cisplatin $(5 \mu \mathrm{M})$ for 5 minutes at room temperature to assess viability. Cells were washed with PBS plus $0.5 \%$ BSA at $500 g$ for 10 minutes. Surface staining was performed by adding a cocktail of preconjugated and homemadeconjugated antibodies (see below) for 30 minutes at room temperature; excess antibodies were removed by washing. Cells were fixed and permeabilized using the FoxP3 Fixation/Permeabilization kit from Invitrogen according to the manufacturer's instructions. Intracellular staining was performed by adding antibodies for 30 minutes at room temperature; excess antibodies were removed by washing. Cells were stained with cell-ID Intercalator-Ir in fixation and permeabilization solution (Fluidigm protocol). Prior to the acquisition, fixed cells were washed with PBS twice and deionized water twice. Cells were resuspended at $5 \times 10^{5}$ per $\mathrm{mL}$ in 1:10 calibration beads (EQFour Element Calibration Beads, Fluidigm), diluted with deionized water and the samples were analyzed with the Helios mass cytometer (Flu- 
idigm) at a flow rate of $0.030 \mathrm{~mL}$ per minute. Mass cytometry data were normalized using MatLab method.

For data analyzing, live $\mathrm{CD} 45^{+} \mathrm{CD}_{11 \mathrm{~b}^{+}} \mathrm{Ly} 6 \mathrm{C}^{\text {hi/lo }}$ cell population was extracted as a new.fcs file using Flowjo and uploaded on Cytobank platform to run unsupervised analysis using the spanning-tree progression analysis of density-normalized events (SPADE) algorithm. The SPADE algorithm generates trees illustrating the relationship between clusters of cells based on a density-dependent downsampling followed by agglomerative clustering to prevent rare cells from being overlooked among the more abundant cellular population.

The following preconjugated and homemade-conjugated primary antibodies were used for surface staining: anti-CD45 (clone HI30, Fluidigm, 89Y-conjugated), anti-CD11c (clone N418, Fluidigm, 209Bi-conjugated), anti-CD4 (clone RM4-5, Fluidigm, 172Yb-conjugated), anti-Ly6C (clone HK1.4, Fluidigm, 162Dy-conjugated), CD45R (B220) (clone RA3-6B2, Fluidigm, 160Gd-conjugated), anti-CD8a (clone 53-6.7, Fluidigm, 153Eu-conjugated), anti-CD3e (clone 145-2C11, Fluidigm, 152Sm-conjugated), anti-Ly6G (clone 1A8, Fluidigm, 151Eu-conjugated), anti-CD19 (clone 6D5, Fluidigm, 149Sm-conjugated), anti-CD11b (clone M1/70, Fluidigm, 148Nd-conjugated), anti-F4/80 (clone BM8, Fluidigm, 146Nd-conjugated), anti-TCRb (clone H57-597, Fluidigm, 143Nd-conjugated), anti-CD69 (clone H1.2F3, Fluidigm, 145Nd-conjugated), anti-CD54 (clone YN1/1.7.4, Fluidigm, 163Dy-conjugated), anti-CD62L (clone MEL-14, Fluidigm, 164Dy-conjugated), anti-CD161 (clone PK136, Fluidigm, 165Ho-conjugated), anti-CD206 (clone C068C2, Fluidigm, 169Tm-conjugated), anti-CD44 (clone IM7, Fluidigm, 171Yb-conjugated), anti-I-A/I-E (clone M5/114.15.2, Fluidigm, 174Yb-conjugated), anti-CD25 (clone 3C7, Fluidigm, 150Nd-conjugated), anti-CCR5 (clone 645807, R\&D Systems, 147Sm-conjugated), anti-GMCSF-R (clone 698423, R\&D Systems, 156Gd-conjugated), anti-TCRgd (clone GL3, Fluidigm, 159Tb-conjugated), anti-CXCR2 (clone 242216, R\&D Systems, 167Er-conjugated), anti-TREM-1 (clone 174031, R\&D Systems, 170Er-conjugated), anti-CXCR1 (clone Q810W6, R\&D Systems, 173Yb-conjugated), anti-CCR2 (clone 475301, R\&D Systems, 175Lu-conjugated), and anti-CX3CR1 (clone 1H14L7, Thermo Fisher Scientific, 176Yb-conjugated). Anti-Foxp3 (clone FJK-16s, Fluidigm, $158 \mathrm{Gd}$-conjugated) antibody was used for intracellular staining.
Statistics. Values are expressed as mean \pm SEM. Differences among values were examined using the nonparametric Mann-Whitney, Kruskal-Wallis tests when appropriate and were considered significant at $P$ less than 0.05 .

Study approval. All animal experiments were conducted according to the guidelines formulated by the European community for experimental animal use (L358-86/609EEC) and were approved by the ethical committee of INSERM and the French Ministry of Agriculture (agreement A75-15-32). The study was conducted according to the Declaration of Helsinki and was approved by the institutional ethics committee (Comité de Protection des Personnes Sud Méditerranée V, protocol 17-030, Collection MCV, ID-RCB: 2017-AO1019-44 and PFS16-007). All enrolled patients gave written informed consent.

\section{Author contributions}

MV conducted experiments, acquired data, analyzed data, performed statistical analysis, and wrote the manuscript. YZ, ISZ, RAR, JJ, AG, LL, BE, FP, JV, A Boufenzer, LG, CG, A Boissonnas conducted experiments, acquired data, analyzed data. PB performed immunostaining in human samples. JR, FL and GC collected human AAA samples and performed qPCR in human aortas. EC provided transgenic mice. SL and LSD provided AF plasma cohort. A Boufenzer and MD provided LR12 peptide. AT, ZM, A Boissonnas, ST, JSS, GC drafted of the manuscript. HAO designed the experiments, analyzed data, performed statistical analysis, drafted of the manuscript and obtained funding for the project. All authors discussed and critically revised the manuscript.

\section{Acknowledgments}

This work was supported by Inserm (ZM, ST, AT, HAO), an ANR program (ANR 2018, ANR-18-CE14-0009-01; to HAO), the FRM (Fondation pour la Recherche Médicale, DEQ20161136699), and the British Heart Foundation (to ZM). We thank Joseph Murcada for his technical assistance.

Address correspondence to: Hafid Ait-Oufella, Inserm U970, Paris Cardiovascular Research Center, 56, rue Leblanc Paris, France. Phone: 3.315.398.8050; Email: hafid.aitoufella@inserm.fr.
1. Nordon IM, et al. Pathophysiology and epidemiology of abdominal aortic aneurysms. Nat Rev Cardiol. 2011;8(2):92-102.

2. Davis FM, et al. Abdominal aortic aneurysm: novel mechanisms and therapies. Curr Opin Cardiol. 2015;30(6):566-573.

3. Mellak S, et al. Angiotensin II mobilizes spleen monocytes to promote the development of abdominal aortic aneurysm in Apoe ${ }^{-/-}$mice. Arterioscler Thromb Vasc Biol. 2015;35(2):378-388.

4. Ye P, et al. GM-CSF contributes to aortic aneurysms resulting from SMAD3 deficiency. JClin Invest. 2013;123(5):2317-2331.

5. Iida Y, et al. Peptide inhibitor of CXCL4CCL5 heterodimer formation, MKEY, inhibits experimental aortic aneurysm initiation and progression. Arterioscler Thromb Vasc Biol. 2013;33(4):718-726.

6. Eliason JL, et al. Neutrophil depletion inhibits experimental abdominal aortic aneurysm forma- tion. Circulation. 2005;112(2):232-240.

7. Bouchon A, et al. Cutting edge: inflammatory responses can be triggered by TREM-1, a novel receptor expressed on neutrophils and monocytes. J Immunol. 2000;164(10):4991-4995.

8. Bouchon A, et al. TREM-1 amplifies inflammation and is a crucial mediator of septic shock. Nature. 2001;410(6832):1103-1107.

9. Tammaro A, et al. TREM-1 and its potential ligands in non-infectious diseases: from biology to clinical perspectives. Pharmacol Ther. 2017;177:81-95.

10. Ford JW, McVicar DW. TREM and TREM-like receptors in inflammation and disease. Curr Opin Immunol. 2009;21(1):38-46.

11. Klesney-Tait J, et al. Transepithelial migration of neutrophils into the lung requires TREM-1. JClin Invest. 2013;123(1):138-149.

12. Boufenzer A, et al. TREM-1 mediates inflammatory injury and cardiac remodeling following myocardial infarction. Circ Res. 2015;116(11):1772-1782.

13. Derive M, et al. Soluble TREM-like transcript-1 regulates leukocyte activation and controls microbial sepsis. J Immunol. 2012;188(11):5585-5592.

14. Derive M, et al. Effects of a TREM-like transcript 1-derived peptide during hypodynamic septic shock in pigs. Shock. 2013;39(2):176-182.

15. Derive M, et al. Attenuation of responses to endotoxin by the triggering receptor expressed on myeloid cells-1 inhibitor LR12 in nonhuman primate. Anesthesiology. 2014;120(4):935-942.

16. Daugherty A, et al. Angiotensin II promotes atherosclerotic lesions and aneurysms in apolipoprotein E-deficient mice. J Clin Invest. 2000;105(11):1605-1612.

17. Zanzinger K, et al. Regulation of triggering receptor expressed on myeloid cells 1 expression on mouse inflammatory monocytes. Immunology. 
2009;128(2):185-195.

18. Dower K, et al. Innate immune responses to TREM-1 activation: overlap, divergence, and positive and negative cross-talk with bacterial lipopolysaccharide. JImmunol. 2008;180(5):3520-3534.

19. Gibot S, et al. A soluble form of the triggering receptor expressed on myeloid cells- 1 modulates the inflammatory response in murine sepsis. JExp Med. 2004;200(11):1419-1426.

20. Swirski FK, et al. Identification of splenic reservoir monocytes and their deployment to inflammatory sites. Science. 2009;325(5940):612-616.

21. Billet S, et al. Gain-of-function mutant of angiotensin II receptor, type $1 \mathrm{~A}$, causes hypertension and cardiovascular fibrosis in mice. JClin Invest. 2007;117(7):1914-1925.

22. Saraff K, et al. Aortic dissection precedes formation of aneurysms and atherosclerosis in angiotensin II-infused, apolipoprotein Edeficient mice. Arterioscler Thromb Vasc Biol. 2003;23(9):1621-1626.

23. Raffort J, et al. Monocytes and macrophages in abdominal aortic aneurysm. Nat Rev Cardiol. 2017;14(8):457-471.

24. Mikolajczyk TP, et al. Role of chemokine RANTES in the regulation of perivascular inflammation, T-cell accumulation, and vascular dysfunction in hypertension. FASEB J. 2016;30(5):1987-1999.

25. Aldasoro Arguinano AA, et al. TREM-1 SNP rs2234246 regulates TREM-1 protein and mRNA levels and is associated with plasma levels of L-selectin. PLoS One. 2017;12(8):e0182226.

26. Johnston WF, et al. Inhibition of interleukin-1 $\beta$ decreases aneurysm formation and progression in a novel model of thoracic aortic aneurysms. Circulation. 2014;130(11 Suppl 1):S51-S59.

27. Xiong W, et al. Blocking TNF-alpha attenuates aneurysm formation in a murine model. J Immunol. 2009;183(4):2741-2746.

28. Freestone T, et al. Inflammation and matrix metalloproteinases in the enlarging abdominal aortic aneurysm. Arterioscler Thromb Vasc Biol. 1995;15(8):1145-1151.

29. Eagleton MJ, et al. Early increased MT1-MMP expression and late MMP-2 and MMP-9 activity during Angiotensin II induced aneurysm formation. J Surg Res. 2006;135(2):345-351.

30. Longo GM, et al. Matrix metalloproteinases 2 and 9 work in concert to produce aortic aneurysms. JClin Invest. 2002;110(5):625-632.

31. Wang Y, et al. TGF-beta activity protects against inflammatory aortic aneurysm progression and complications in angiotensin II-infused mice. J Clin Invest. 2010;120(2):422-432.

32. Curci JA, et al. Expression and localization of macrophage elastase (matrix metalloproteinase-12) in abdominal aortic aneurysms. J Clin Invest. 1998;102(11):1900-1910.

33. Newby AC. Metalloproteinase expression in monocytes and macrophages and its relationship to atherosclerotic plaque instability. Arterioscler Thromb Vasc Biol. 2008;28(12):2108-2114.

34. Newby AC. Metalloproteinases promote plaque rupture and myocardial infarction: A persuasive concept waiting for clinical translation. Matrix Biol. 2015;44-46:157-166.

35. Gibot S. Clinical review: role of triggering receptor expressed on myeloid cells-1 during sepsis. Crit Care. 2005;9(5):485-489.

36. Wang YS, et al. TREM- 1 is a positive regulator of TNF- $\alpha$ and IL-8 production in U937 foam cells. Bosn J Basic Med Sci. 2012;12(2):94-101.

37. Lo TH, et al. TREM-1 regulates macrophage polarization in ureteral obstruction. Kidney Int. 2014;86(6):1174-1186.
38. Nakashima T, et al. TLR4 is a critical regulator of angiotensin II-induced vascular remodeling: the roles of extracellular SOD and NADPH oxidase. Hypertens Res. 2015;38(10):649-655.

39. Wang YD, et al. Pharmacological therapy of abdominal aortic aneurysm: an update. Curr Vasc Pharmacol. 2018;16(2):114-124.

40. Li H, et al. Modulation of immune-inflammatory responses in abdominal aortic aneurysm: emerging molecular targets. J Immunol Res. 2018;2018:7213760.

41. Cuvier V, et al. A first-in-man safety and pharmacokinetics study of nangibotide, a new modulator of innate immune response through TREM-1 receptor inhibition. Br J Clin Pharmacol. 2018;84(10):2270-2279.

42. Francois B, et al. Nangibotide in patients with septic shock: a Phase 2a randomized controlled clinical trial. Intensive Care Med. 2020;46(7):1425-1437.

43. Lareyre F, et al. Investigation of plasma inflammatory profile in diabetic patients with abdominal aortic aneurysm: a pilot study. Vasc Endovascular Surg. 2018;52(8):597-601.

44. Lareyre F, et al. Association of abdominal aortic aneurysm diameter with insulin resistance index. Biochem Med (Zagreb). 2018;28(3):030702.

45. Joffre J, et al. Genetic and pharmacological inhibition of TREM-1 limits the development of experimental atherosclerosis. JAm Coll Cardiol. 2016;68(25):2776-2793.

46. Ponnuswamy P, et al. Angiotensin II synergizes with BAFF to promote atheroprotective regulatory B cells. Sci Rep. 2017;7(1):4111.

47. Kolachala VL, et al. Loss of L-selectin-guided $\mathrm{CD}^{+}$, but not $\mathrm{CD}^{+}$, cells protects against ischemia reperfusion injury in a steatotic liver. Hepatology. 2017;66(4):1258-1274. 